\title{
Enzymatic Coupled Mechanical Defibrillation Process for the Production of Corn (Zea Mays) Cob Microfibrillated Cellulose: Preparation, Characterization and Evaluation as Pickering Emulsifier for Oil-In-Water Emulsion
}

\section{Teck-Kim Tang \\ Yee-Ying Lee \\ Monash Universiti Malaysia \\ Eng-Tong Phuah \\ Universiti Tunku Abdul Rahman \\ Chin-Ping Tan \\ Universiti Putra Malaysia}

Institute of Bioscience, Universiti Putra Malaysia

Sivaruby Kanagaratnam

Malaysia Palm Oil Board

Yong Wang

Jinan University

Ling-Zhi Cheong

Ningbo University

Ying Li

Jinan University

Nurul Aini Jamalullail

Institute of Bioscience, Universiti Putra Malaysia

Oi-Ming Lai ( $\square$ omlai@upm.edu.my )

Universiti Putra Malaysia https://orcid.org/0000-0003-1205-149X

\section{Research Article}

Keywords: nanocellulose, emulsion, rheology, stability, corn cob

Posted Date: March 12th, 2021

DOI: https://doi.org/10.21203/rs.3.rs-282587/v1 
License: (c) (i) This work is licensed under a Creative Commons Attribution 4.0 International License. Read Full License 


\section{Enzymatic coupled mechanical defibrillation process for the production of corn (Zea mays) cob microfibrillated cellulose: preparation, characterization and evaluation as Pickering emulsifier for oil-in-water emulsion}

\section{Authors}

Teck-Kim Tang a , Yee-Ying Lee ${ }^{\text {b,c }}$, Eng-Tong Phuah ${ }^{\mathrm{d}}$, Chin-Ping Tan ${ }^{\mathrm{e}}$, Sivaruby Kanagaratnam ${ }^{\mathrm{f}}$, Yong Wang ${ }^{\mathrm{g}}$, Ling-Zhi Cheong ${ }^{\mathrm{h}}$, Ying Li ${ }^{\mathrm{g}}$, Nurul Aini Jamalullail $^{\text {a }}$, Oi-Ming Lai ${ }^{\text {a, }}{ }^{*}$

\section{Affiliations}

${ }^{a}$ International Joint Laboratory on Plant Oils Processing and Safety (POPS) JNU-UPM, Institute of Bioscience, Universiti Putra Malaysia, 43400, Serdang, Selangor.

b Monash-Industry Palm Oil Education and Research Platform, Monash University Malaysia, 47500 Bandar Sunway, Selangor, Malaysia.

${ }^{c}$ School of Science, Monash University Malaysia, 47500 Bandar Sunway, Selangor, Malaysia.

${ }^{\mathrm{d}}$ Department and Agriculture and Food Science, Universiti Tunku Abdul Rahman, 31900 Kampar, Perak.

e International Joint Laboratory on Plant Oils Processing and Safety (POPS) JNU-UPM, Department of Food Technology, Faculty of Food Science and Technology, Universiti Putra Malaysia, 43400 UPM Serdang, Selangor, Malaysia

${ }^{\mathrm{f}}$ Food Technology and Nutrition Unit, Malaysian Palm Oil Board, 6 Persiaran Institusi, Bandar Baru Bangi, 43000 Kajang, Selangor, Malaysia.

${ }^{\mathrm{g}}$ International Joint Laboratory on Plant Oils Processing and Safety (POPS) JNU-UPM, Department of Food Science and Engineering, College of Science and Engineering, Jinan University, Guangzhou 510632, China.

${ }^{\mathrm{h}}$ Department of Food Science, School of Marine Science, Ningbo University, Fenghua Road 818, Ningbo, P.R. China

${ }^{\text {i }}$ Department of Bioprocess Technology, Faculty of Biotechnology and Biomolecular Sciences, Universiti Putra Malaysia, 43400 UPM Serdang, Selangor, Malaysia.

\section{*Corresponding author}

E-mail address: omlai@upm.edu.my 
41 Microfibrillated cellulose (MFC) is a type of nanocellulose having multiple 42 functionalities. Typically, MFC was produced from mechanical high pressure 43 homogenization process. However, this process is energy intensive and the fibrous nature 44 of MFC often causes instrument blockage. The present study aims to utilize 45 endoglucanse enzyme as environmentally friendly approach to pretreat fiber structure 46 prior to undergoing mechanical defibrillation for the production of MFC from corn cob. 47 Alkaline and bleached pretreated corn cob was treated with endoglucanase Fibercare $\mathrm{R}$ 48 from $0 \%$ to $2.5 \%$ before passing through high pressure homogenizer. It was found that 49 incorporation of $0.02 \%$ of endoglucanase was sufficient to soften the corn cob cellulose 50 and further prevent the blockage of homogenizer. Subsequently, the $0.02 \%$ endoglucanse 51 treated corn cob was passed through different cycles of homogenization from 0 cycle to 5210 cycle for MFC production. It was observed that the water retention, zeta potential and 53 shear viscosity of the MFC increases with homogenization cycle. MFC produced had a 54 gel like consistency. Next, emulsifying stabilizing properties of MFC produced from 55 cycle 0 to cycle 10 as well as their amount from $0 \%$ to $1 \%$ were also assessed. Increase 56 in homogenization cycle and the amount of MFC promote emulsion stability as observed 57 from the low creaming index which is mainly attributed to the high shear viscosity and 58 G'G' ' crossover of the emulsion. In all, the MFC derived from corn cob via enzymatic 59 coupled with high pressure homogenization process has the potential to be used as gel 60 like stabilizer in oil-in-water food emulsion system.

61 Keywords: nanocellulose, emulsion, rheology, stability, corn cob

\section{Introduction}

63 Microfibrillated cellulose (MFC) is a purified form of cellulose that is isolated from 64 cellulose fiber having a high aspect ratio with few nanometer in diameter and several 65 microns in length. When suspended in water, MFC creates a strong three-dimensional 66 network structure that is able to give rise to viscous-gel like properties. Attributed to its 67 sustainable and multifunctional properties, MFC received a lot of attention for used in 68 various applications in electronic, biomedical and food industries. Traditionally, MFC 69 was produced from softwood pulp. However, today, a lot of attention focuses on the use 70 of herbaceous plant or agricultural waste materials for MFC production due to 71 sustainability issue. Herbaceous plant offer more advantages over softwood as it is 
72 readily available, sustainable, and possesses simple structural make up than wood. Thus,

73 herbaceous plan requires a lesser and simpler pretreatment process to disintegrate and

74 defibrillate the fiber during the production of MFC (Sedjo and Lyon 2015; Trache et al.

75 2017).

76 Often, MFC were prepared using mechanical shearing approach such as high pressure 77 homogenization to defibrillate cellulose bundle into filament form. Winuprasith \& 78 Suphantharika (2013) employed high pressure homogenization process to produce MFC 79 from mangosteen skin. However, high emulsification process has its setbacks. It is often 80 regarded as an energy intensive process and frequently causes blockage or breakdown of 81 high pressure homogenizer especially when the sample treated is hard in texture or has 82 uneven particle size (Henriksson et al. 2007). As a result, upscale and commercialize of 83 MFC produced via high pressure homogenization approach is considered expensive and 84 a challenging task.

85 An emulsion is a mixture of two or more liquids that are immiscible. Conventionally, 86 emulsion was stabilized by stabilizers that is mainly derived from surfactants. 87 Nevertheless, emulsion can also be stabilized by solid particles and the emulsion formed 88 is known as 'Pickering emulsions' (Pickering 1907). Example of solid particles that can 89 be utilized as Pickering emulsifier include: hydrophobized fumed silica (Frelichowska et 90 al. 2010), food-grade particles like protein, fat crystal, and polysaccharide complexes 91 (Tavernier et al. 2016), bacterial cellulose nanocrystals (Kalashnikova et al. 2011). 92 Particle stabilized emulsion offers several advantages than those prepared from 93 surfactants. Pickering emulsion offers to be a good source in replacing or reducing the 94 usage of emulsifier that may often cause adverse health effects such as irritancy. 95 Furthermore, emulsion stabilized by solid particle is extremely stable against coalescence. 96 Apart from the above mentioned particles, nanocellulose such as MFC or nanocrystal 97 cellulose was the recent particle used for stabilizing emulsion. For instance, study 98 performed by Winuprasith et al (2013) utilized $0.7 \%$ of nanocellulose from mangosteen 99 rind to stabilize a $30 \%$ and $10 \% \mathrm{w} / \mathrm{w}$ soybean oil emulsion system. The study revealed 100 that MFC demonstrated the ability to restrict the movement of emulsion droplets and 101 prevent the dispersed phase from coalescing in oil-in-water emulsion system 102 (Winuprasith and Suphantharika 2013). Unlike other solid stabilizers, cellulose based 103 stabilizer possesses extra attributes because of it zero calorie characteristic (Anderson 104 and Eastwood 1989; Slavin 2005). This property further extended the utilization of MFC 
105 in food industries as low calorie stabilizer in developing healthy low or reduced calorie

106 food emulsion products.

107 Corn is one of the few important cereal crops after wheat and rice that is utilized widely 108 as staple food worldwide. Out of total corn production, there is around $15 \%$ of waste 109 being generated from corn processing in the form of corn cob (Gradinaru et al. 2018). 110 Today, majority of the corn cob produced is widely used as heat generator, animal 111 bedding, oil sorbents, polishing agents, biofuel and activated carbons. Nevertheless, corn 112 cob is a rich reservoir of carbohydrates (Maha et al., 2010). Study found that corn cob 113 consists mainly of carbohydrate in the form of $38.8 \%$ cellulose, $44.4 \%$ hemicellulose and 114 11.9\% lignin (Pointner et al. 2014). Furthermore, it has relatively low impurities since it 115 is covered with husk and corn kernel.

116 Therefore, the present study aims to explore the transformation of underutilized corn cob 117 waste into value added MFC that can be used as rheological modifier to stabilize oil-in118 water emulsion system using an improved and milder high pressure homogenization 119 approach via the aid of enzyme. The current work investigated the facilitation of 120 endoglucanse Fibercare R to defibrillate fiber bundle for the production of MFC using 121 high pressure homogenizer to resolve the blockage issue of high pressure homogenize. In 122 the present study, the effect of different amount of endoglucanse enzyme ( $0 \%$ to $2.5 \%$ ) 123 on the MFC properties produced as well as the tendency of blockage of homogenizer was 124 evaluated. Subsequently, the current work also investigate the influence of the cycles of 125 homogenization (cycle 0 to cycle 10) and amount of MFC (o\% to 1\%) in stabilizing oil126 in-water emulsion.

\section{Materials and Methods}

\section{$128 \quad 2.1$ Materials}

129 Corn cob was obtained from local corn supplier Nelson's Franchise (M) Sdn Bhd (Shah 130 Alam, Selangor, Malaysia). The raw corn cob consisted of $43.8 \%$ cellulose and $47.7 \%$ 131 hemicellulose, respectively. It was ground using coconut grater machine and 132 subsequently pressed using coconut milk separator to remove the juice from the corn cob. 133 Subsequently, the corn cob was dried in an oven at $55^{\circ} \mathrm{C}$ and sieved through $1 \mathrm{~mm}$ mesh 134 size sieve. Pulpzyme HC (xylanase) and Fibercare R were purchased from Novozyme 135 (Bagsvaerd, Denmark). Palm olein (Buruh, Malaysia) with IV 56 was purchased from 136 local hypermarket. All the reagents and chemicals inclusive of sulphuric acid (Merck, 
137 USA) potassium permanganate (Merck, USA), sodium hydroxide (Merck, USA) and 138 sodium hypochlorite (Merck, USA) used were of analytical grade.

\subsection{Production of MFC}

140 Alkaline pretreatment was performed to remove the hemicellulose from corn cob. A $6 \%$ 141 (w/w) dried corn cob was treated with $0.5 \mathrm{M}$ sodium hydroxide for $30 \mathrm{~min}$ at $80{ }^{\circ} \mathrm{C}$. After 142 alkaline treatment, the corn cob suspension was filtered and washed thoroughly with 143 ultrapure water. Alkaline treated corn cob was then treated with Pulpzyme HC. Pulpzyme $144 \mathrm{HC}$ of $0.03476 \%(\mathrm{v} / \mathrm{v})$ was added to the $3 \%$ alkaline treated corn cob that was suspended 145 in buffer solution containing $0.11 \mathrm{M} \mathrm{KH}_{2} \mathrm{PO}_{4}$ and $0.09 \mathrm{M} \mathrm{Na} \mathrm{HPO}_{4}$. After $45 \mathrm{~min}$, the 146 corn cob fiber was treated with endoglucanse Fibercare $\mathrm{R}$ of varies concentration from $1470 \%, 0.02 \%, 0.10 \%, 0.5 \%$ to $2.5 \%$ (denoted as G0, G0.02, G0.10, G0.50 and G2.50) for 2 148 hours. Endoglucanse Fibercare R was used for softening the corn cob to ease the fiber 149 passage through the subsequent high pressure homogenization process used for the 150 production of MFC. The enzymatic reaction was terminated by heating the suspension at $15180{ }^{\circ} \mathrm{C}$ for $30 \mathrm{~min}$. Then, bleaching was performed to remove lignin from the treated corn 152 cob. The pretreated corn cob were bleached with $0.6 \%$ sodium hypochlorite at $80{ }^{\circ} \mathrm{C}$ for 1532 hours and the filtrate were washed thoroughly with ultrapure water. Next, a $0.5 \%$ of the $1540.02 \%$ endoglucanase treated corn cob were homogenized using high pressure 155 homogenizer (HPH) (Panda 2K, Niro Soavi, Deutschland, Lubeck, Germany) at 1000 bar 156 at different passes consisting of $0,2,4,6,8$ to 10 , respectively to produce MFC. In order 157 to evaluate the addition of Fibercare $\mathrm{R}$ in easing the homogenization process, any 158 blockage in the high pressure homogenizer were recorded based on observation. 159 Blockage was considered when fiber suspension were not able to pass through high 160 pressure homogenizer. Alkaline and bleaching pretreatment managed to successfully 161 removed hemicellulose and lignin giving rise to MFC with $78.9 \%$ of cellulose and $14.9 \%$ 162 of hemicellulose content.

\subsection{Characterization of MFC}

\subsubsection{Degree of polymerization}

165 The degree of polymerization of MFC and raw corn cob were determined according to 166 ISO 5351:2012 method and calculated from the intrinsic viscosities at $25{ }^{\circ} \mathrm{C}$ using the 167 equation (1): 
where $\eta$ represents intrinsic viscosity and DP represents degree of polymerization

\section{$170 \quad$ 2.3.2 Stability}

171 Stability index and water holding capacity of MFC were evaluated using optical 172 centrifuge analyzer (Lumifuge 114 LUM GmbH, Berlin, Germany) with a $2 \mathrm{~mm}$ path 173 length rectangular sample tubes. MFC was centrifuged at $2000 \mathrm{rpm}$ for $127.5 \mathrm{~min}$ at $17425^{\circ} \mathrm{C}$. Stability index was calculated based on equation 2 :

$$
\frac{\text { length of low trasmision area (MFC area) }}{\text { Total sample length }} \times 100
$$

Water holding capacity of MFC produced were calculated using equation 3:

$$
\frac{\text { Stability index }}{\text { MFC dried weight } \times 100} \times \text { volume }
$$

Zeta potential of MFC were measured using Zetasizer Nano ZS (Malvern Instruments, 179 Malvern, UK) via a disposable folded capillary cells (DTS 1060) at $25^{\circ} \mathrm{C}$.

180 Water evaporation test was carried out by incubating the MFC at $60{ }^{\circ} \mathrm{C}$ for a duration of one week. The percentage of water lost was calculated using equation 4:

$$
\frac{\text { initial weight-final weight }}{\text { inital weight }} \times 100
$$

\subsubsection{Morphology}

184 Surface morphology of the MFC were examined with a JEOL 6400V scanning electron microscope (JEOL USA, Inc., Peabody, MA, USA). MFC were freeze dried and subsequently affixed to an aluminum stub using carbon filled tape. The assembly was then coated with gold before examine under electron microscope.

\subsubsection{Rheology}

189 Rheological properties of the freshly prepared MFC suspensions were measured using a 190 rheometer (Thermo Scientific HAAKE Rheostress 6000 Universal Rheometer, 191 Buckinghamshire, Germany) equipped with a $60 \mathrm{~mm}$ diameter titanium plate-and-plate 192 with universal temperature controller. A $2.9 \mathrm{ml}$ of sample was rest for 5 minutes on 193 peltier plate to erase additional stress as well as to equilibrate the temperature. Shear viscosity was monitored by increasing the shear rate from 0.1 to $300 \mathrm{~s}^{-1}$ at $25^{\circ} \mathrm{C}$. A linear 
195 viscoelastic range (LVR) was determined with a strain sweep at frequency of $1 \mathrm{~Hz}$ from

$1960.01 \mathrm{~Pa}$ until $100 \mathrm{~Pa}$ for dynamic viscoelastic measurements. The determined LVR was

197 subsequently used for the frequency sweep. A dynamic frequency sweep was conducted

198 by applying a constant strain of $0.1 \mathrm{~Pa}$ (determined by LVR) which was within the linear

199 region over a frequency range between $0.1 \mathrm{~Hz}$ and $10 \mathrm{~Hz}$.

\subsection{MFC stabilized oil-in-water emulsion}

201 MFC obtained under HPH cycles of 0, 2, 4, 6, 8 and 10 cycles (denoted as C0, C2, C4, 202 C6, C8 and C10) were utilized to stabilize oil-in-water emulsions. The emulsions were 203 prepared by pre-mixing 9 parts of $0.5 \%$ MFC with 1 part palm olein (w/w) using rotor204 stator (Silverson L4R, Buckinghamshire, UK) set at $7000 \mathrm{rpm}$ for 5 minutes The coarse 205 dispersions were then passed through high pressure homogenizer (Panda $2 \mathrm{~K}$, Niro Soavi, 206 Deutschland, Lubeck, Germany) at 500 bar for 3 passes. The MFC produced at cycle 10 207 were also used to stabilize oil-in-water emulsions at varying concentration ranging from $2080.00 \%$ to $1.00 \%(\mathrm{w} / \mathrm{w})$ in order to assess the influence of MFC amount on the emulsion 209 stability.

\section{$210 \quad 2.5$ Characterization of the MFC stabilized emulsion}

\section{$211 \quad$ 2.5.1 Particle size}

212 The particle sizes of the emulsions were analyzed using Mastersizer 2000 instrument 213 (Malvern Instruments Ltd, Worcestershire, UK). Emulsions were diluted to around 0.05\%

214 (w/w) with distilled water to prevent multiple scattering effects. Subsequently, samples

215 were dispersed in distilled water at $1200 \mathrm{rpm}$ until obscuration rate of $15 \%$ was obtained 216 (Laca et al. 2010). The reflective index of dispersant (water) and disperser (emulsion) 217 were set at 1.33 and 1.46, respectively. The particle size parameters were recorded as 218 follow: volume-weighted mean diameter $d_{4,3}$, surface weighted mean diameter $\mathrm{D}_{3,2}$ span 219 index-quantification distribution width: (D90 - D10) / D50, D10, D50, and D90 220 (cumulative volume of particle sizes that make up of emulsion volume of $10 \%, 50 \%$, and $22190 \%)$.

\section{$222 \quad$ 2.5.2 Stability}

223 The stability of emulsions under accelerated conditions were tested using optical 224 centrifuge analyzer (Lumifuge 114 LUM GmnH, Berlin, Germany) with $2 \mathrm{~mm}$ path 
225 length rectangular sample tubes. Emulsions were centrifuged at $1000 \mathrm{rpm}$ for $127.5 \mathrm{mins}$

226 (representing 255 cycles) at 3 different temperatures of $5{ }^{\circ} \mathrm{C}, 25{ }^{\circ} \mathrm{C}$ and $45{ }^{\circ} \mathrm{C}$,

227 respectively to evaluate its stability when stored under different temperature conditions.

228 Creaming index were calculated as equation (6):

$$
(C I) \%=\frac{\text { final position of bewteen clear phase \& creaming layer }}{\text { Total sample height }} \times 100 \text {------(6) }
$$

\subsubsection{Rheology}

231 The rheological properties of freshly prepared emulsions which include shear viscosity 232 and dynamic frequency sweep were evaluated using rheometer (Rheostress 6000 Haake, 233 Buckinghamshire, Germany) coupled with $60 \mathrm{~mm}$ plate to plate probe. The shear 234 viscosity and dynamic frequency sweep test were set in accordance to Section 2.3.6. 235 Additional thixotropy measurements on the emulsion were also conducted. The degree of 236 thixotropy was assessed based on the area difference of the hysteresis loops. Hysteresis 237 loop was generated from $0 \mathrm{~s}^{-1}$ to $300 \mathrm{~s}^{-1}$ for 5 mins then immediately returned to $0 \mathrm{~s}^{-1}$.

\section{$238 \quad 2.5 .4$ Morphology}

239 The morphology of the emulsion samples prepared from C2 and C10 were visualized 240 using the JEM-2100F field emission analytical electron microscope (JOEL, Tokyo, 241 Japan). A drop of the emulsion was placed onto a 400-mesh formvar carbon film-coated 242 copper grid and the excess solution was blotted with filter paper. Then, the grid was 243 negatively stained by uranyl acetate (Meena et al. 2012). The excess solution was blot 244 with filter paper. The grid were then dried for 15 mins prior to visualization.

\section{$245 \quad 2.6$ Statistical analysis}

246 All measurements were performed in triplicates unless stated. Significant differences $247(\mathrm{P}<0.05)$ of the samples were analyzed using one way analysis of variance (ANOVA) by 248 LSD test. Results are expressed as mean values \pm standard deviations.

\section{Results and discussions}

\section{$250 \quad 3.1$ Corn cob pretreated with endoglucanse}

251 Corn cob fiber was treated with $0 \%, 0.02 \%, 0.1 \%, 0.5 \%$ to $2.5 \%$ of Fibercare R. As an 252 endoglucanse enzyme, the Fibercare R enables softening of corn cob fiber that ease its 253 passage through high pressure homogenizer thus preventing the blockage or breakdown 
254 of the equipment. Table 1 shows that endoglucanse treatment from $0.02 \%$ to $2.5 \%$ on

255 blockage of high pressure homogenizer. Incorporation of the endoglucanase enables the

256 fiber suspension to pass through the high pressure homogenizer smoothly without

257 causing any blockage whilst those without endoglucanase treatment show to block the

258 high pressure homogenizer. Also, endoglucanse pretreatment reduces the degree of

259 polymerization of the fiber. Study shows that the reduction in the degree of

260 polymerization was the smallest when corn cob fiber was treated with $0.02 \%$ of

261 endoglucanase whilst a significant $(\mathrm{P}<0.05)$ reduction in the degree of polymerization

262 was observed when corn cob was treated with $2.5 \%$ of endoglucanse enzyme. It should

263 be highlighted that fiber with low degree of polymerization is undesirable for MFC

264 production as it lead to the formation of microcrystalline cellulose instead.

265 Table 1: Degree of Polymerization (DP) and frequency of high pressure homogenizer blocked by 266 fiber

\begin{tabular}{ccc}
\hline Fiber & Total number of HPH blocked within 10 tests & DP \\
\hline G0.0 (Control) & 2 & $991 \pm 4^{\mathrm{a}}$ \\
G0.02 & 0 & $934 \pm 4^{\mathrm{b}}$ \\
G0.1 & 0 & $735 \pm 3^{\mathrm{c}}$ \\
G0.5 & 0 & $717 \pm 5^{\mathrm{d}}$ \\
G2.5 & 0 & $688 \pm 7^{\mathrm{e}}$
\end{tabular}

267 Data were performed in triplicate. Mean \pm standard deviation values in the same column followed by 268 different letters are significantly different $(\mathrm{p}<0.05)$.

\section{$269 \quad 3.2$ Characteristic of MFC}

270 Since $0.02 \%$ of endoglucanse enzyme was sufficient to prevent blockage of high pressure

271 homogenizer while still retain a high degree of polymerization, it was selected to treat the

272 corn con fiber to soften the texture before defibrillation process performed by high

273 pressure homogenizer. However, corn cob fiber suspension treated with $0.02 \%$ of

274 endoglucanse tend to sediment to the bottom instead of dispersing well in the suspension.

275 MFC that is well dispersed is important to act as stabiliser for oil-in-water emulsions.

276 Therefore, high pressure homogenization was used to improve the stability of the MFC

277 suspension. Endgolucanse treated corn cob was defibrillate using high pressure

278 homogenizer from cycle 0 to cycle 10. 
280 It was found that homogenization successfully transform the MFC from liquid 281 suspension into a gel like suspension. MFC tends to absorb water after undergoing 282 defibrillation process using high pressure homogenizer which leads to the formation of 283 gel like consistency. Stability of MFC gel increased in tandem with the number of cycles 284 of high pressure homogenization and reached a plateau state after homogenizing for 6 285 cycles. The MFC produced at C10 was the most stable indicated by the highest water 286 holding capacity of $12763 \%$ that represents the absorption of 127.63 gram of water per 287 gram of sample.

288 The water holding capacity properties of MFC was further confirmed by the \% of water 289 evaporation or water loss due to evaporation. It was found that the water evaporation 290 from MFC reduced with high homogenization cycles. Without homogenization, water 291 evaporation of MFC was 52.42\%. Homogenization for 10 cycle reduced the water 292 evaporation of MFC to 34.90\%. A reduction in water evaporation after homogenization 293 could be attributed to the increase in the defibrillation process that breaks the fiber along 294 the lateral direction thus making it become thinner. Defibrillation successfully exposed 295 the hydroxyl side groups of MFC further attract the binding of water molecules to MFC 296 via hydrogen bonding. Also, the amount of MFC released from fiber bundle increases 297 after homogenization which restricted the movement of water molecule making it to have 298 gel like texture. The gel like properties of MFC makes it an excellent water stabilizing or 299 wetting and control release agent.

300 Stability of the MFC was also verified by evaluating its zeta potential. Typically, samples 301 with zeta potential less $-30 \mathrm{mV}$ or higher than $+30 \mathrm{mV}$ are considered to be stable as the 302 value was sufficient for the molecules to repel each other preventing them from 303 coalescene. Table 2 shows the zeta potential of the MFC. Zeta potential of MFC 304 increased with HPH cycles. Control without passing through high pressure homogenizer 305 possess zeta potential of $\sim 23.20 \mathrm{mV}$ while MFC produced at cycle 10 attained zeta 306 potential of $\sim 30 \mathrm{mV}$. All the MFC have zeta potential value were lower than $\sim 30 \mathrm{mV}$ 307 regardless of the number of cycles of homogenization demonstrating that the MFC 308 produced are relatively stable.

309 Table 2: Degree of polymerization (DP), water holding capacity (WHC), stability, evaporation and 310 zeta potential of microfibrillated cellulose treated with $\mathbf{0 . 0 2 \%}$ of endoglucanase after undergoing 311 different cycles of homogenization 


\begin{tabular}{llllll}
\hline \multicolumn{5}{c}{ Analysis performed } \\
\hline Cycle & DP (unit) & WHC $(\mathrm{g} / \mathrm{g})$ & Stability $(\%)$ & Evaporation $(\%)$ & Zeta potential (mV) \\
\hline C0 & $934 \pm 4^{\mathrm{a}}$ & $36.51 \pm 0.21^{\mathrm{a}}$ & $18.18 \pm 0.01^{\mathrm{a}}$ & $52.42 \pm 2.66^{\mathrm{a}}$ & $-23.20 \pm 0.26^{\mathrm{a}}$ \\
$\mathrm{C} 2$ & $894 \pm 5^{\mathrm{b}}$ & $80.56 \pm 0.11^{\mathrm{b}}$ & $40.00 \pm 0.20^{\mathrm{a}}$ & $46.86 \pm 2.88^{\mathrm{ab}}$ & $-33.63 \pm 0.90^{\mathrm{b}}$ \\
C4 & $864 \pm 8^{\mathrm{c}}$ & $97.56 \pm 1.54^{\mathrm{c}}$ & $47.95 \pm 0.46^{\mathrm{c}}$ & $47.09 \pm 3.25^{\mathrm{ab}}$ & $-36.53 \pm 1.11^{\mathrm{b}}$ \\
C6 & $838 \pm 1^{\mathrm{d}}$ & $121.39 \pm 3.78^{\mathrm{d}}$ & $60.45 \pm 0.40^{\mathrm{d}}$ & $42.90 \pm 1.09^{\mathrm{b}}$ & $-36.07 \pm 4.50^{\mathrm{b}}$ \\
C8 & $738 \pm 4^{\mathrm{e}}$ & $124.56 \pm 1.29^{\mathrm{d}}$ & $61.59 \pm 0.41^{\mathrm{d}}$ & $31.35 \pm 2.33^{\mathrm{c}}$ & $-30.67 \pm 0.32^{\mathrm{b}}$ \\
C10 & $729 \pm 2^{\mathrm{e}}$ & $127.63 \pm 0.55^{\mathrm{d}}$ & $62.73 \pm 0.46^{\mathrm{d}}$ & $34.90 \pm 5.89^{\mathrm{c}}$ & $-36.00 \pm 3.14^{\mathrm{b}}$
\end{tabular}

312 Data were performed in triplicate. Mean \pm standard deviation values in the same column followed by 313 different letters are significantly different $(\mathrm{p}<0.05)$.

\section{$314 \quad$ 3.2.2 Microstructure}

315 Figure 1 A-F shows the microstructure of MFC observed under SEM. Prior to 316 undergoing homogenization the corn cob fiber retain much of their intact structure 317 (Figure 1A). After high pressure homogenization, corn cob fiber bundle disintegrated 318 into filaments. The filaments entangled into a three dimensional network-like after 319 undergoing homogenization for 6-10 cycles (Figure 1 B -F). Homogenization also 320 reduced the diameter of the MFC. It was estimated that the diameter of MFC observed 321 under 15000x resolution was around 50nm as shown in Figure $1 \mathrm{~F}$.

A

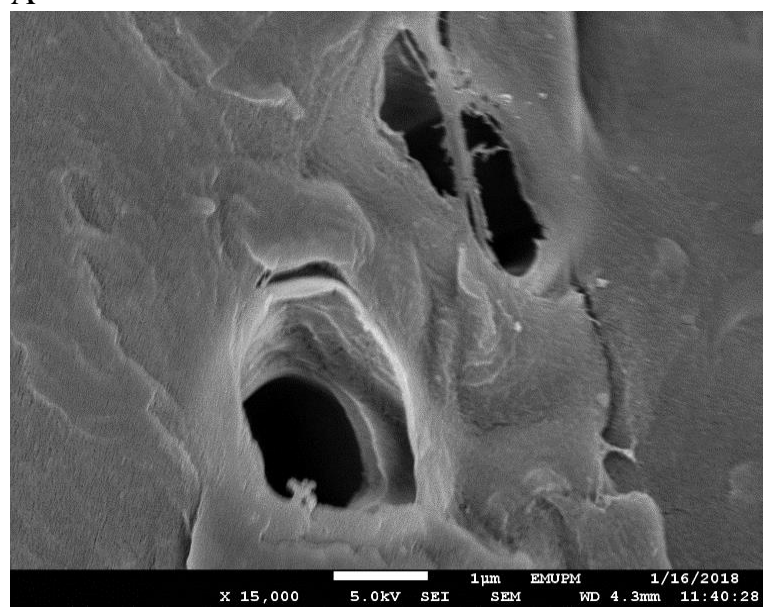

B

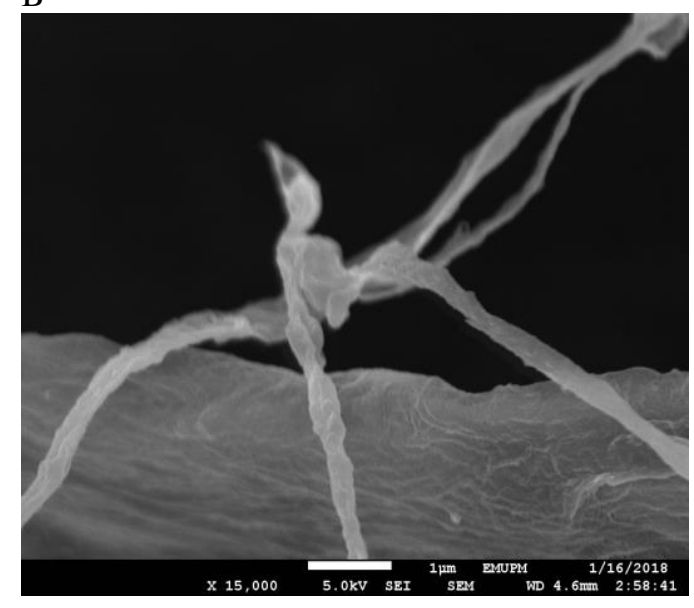



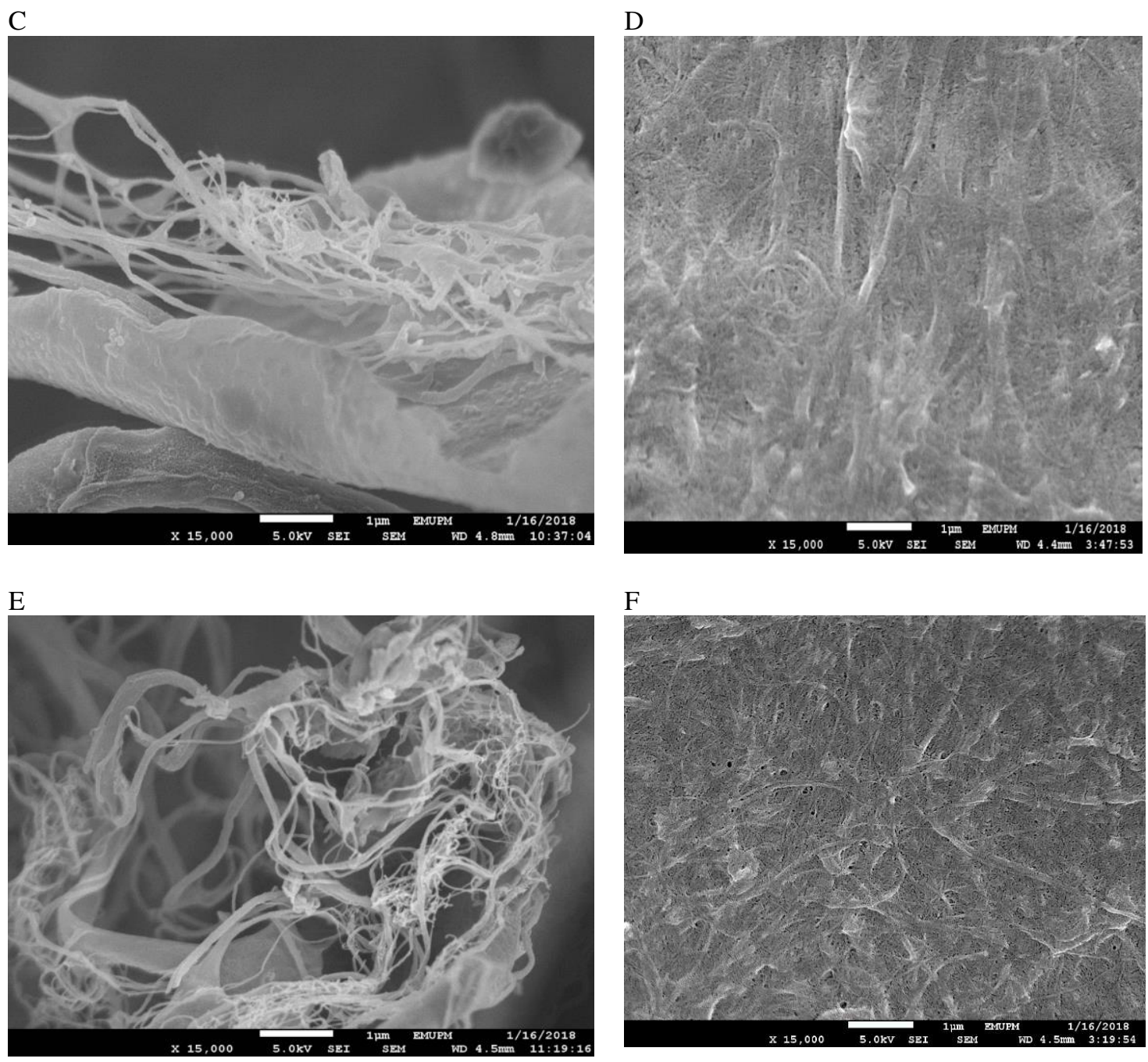

Figure 1: SEM photograph of MFC produced from different cycles of HPH.

\section{$326 \quad$ 3.2.3 Rheology}

327 Figure 2A shows the shear viscosity of MFC produced from cycle 0 to cycle 10 of 328 homogenization. All the MFC samples showed shear thinning/pseudoplastic behavior.

329 Shear viscosity decreased from around 50 Pa.s to 0.01 Pa.s when the shear rate increased 330 from 0.1 to 300 1/s. C2 had the lowest shear viscosity. Viscosity of MFC increased with 331 high pressure homogenization cycle. Increase in homogenization cycle resulted in 332 disintegration of the fiber and thus releasing more MFC from the fiber bundle. Attributed 333 to the high aspect ratio, MFC as such creates a network-like structure which can resist 334 shear force. 
335 Figure 2B shows the shear viscosity at log scale to assess the flat plateau region at low shear rate used for the estimation of zero shear viscosity marked as X. Zero shear viscosity gave the indication of molecular weight distribution. A substance with a broad molecular weight distribution (MWD) will have shorter zero shear plateau region and vice versa. Figure $2 \mathrm{~B}$ illustrates that $\mathrm{C} 4$ and $\mathrm{C} 6$ had a border molecular weight distribution. It can be inferred that $\mathrm{C} 4$ and $\mathrm{C} 6$ contain a mixture of different molecular structure of the cellulose either in the form of bundles or cellulose thread which might be due to the partial released of fiber from the fiber bundles (Figure $1 \mathrm{C}-\mathrm{D}$ ). Meanwhile, C2 and C10 samples had a narrow molecular weight distribution. C2 mainly contained similar large sized fiber bundle (Figure $1 \mathrm{~B}$ ) whilst C10 has almost all its cellulose converted to MFC (Figure $1 \mathrm{~F}$ ). Thus, the molecular structure of cellulose in this two situations is homogenous.

347 Yield stress behavior is an important feature for gel-like or semisolid-like substance. A 348 yield stress is the minimum stress required for a material to initiate to flow. Prior to this, sample usually exhibit elastic deformation or simply solid-like property. A high stress value indicated that more stress needs to be applied for the sample to flow. As shown in 351 Figure 2C, cycle 10 has the highest yield stress while cycle 2 has the lowest yield stress 352 indicating that MFC gel produced at cycle 10 is more stable and stiffer than MFC gel 353 produced at cycle 2.

354 Figure 3 A-B shows amplitude sweep tests of MFC produced under cycle 0 to cycle 10 of high pressure homogenization: G' storage modulus (elastic Component) and G', loss modulus (viscous component) vs oscillation stress (frequency fixed at $1 \mathrm{~Hz}$ ). Viscoelastic material usually exhibit linear viscous region where $G^{\prime}>G^{\prime}$ '. It is independent to stress until the structure breaks down and the material switches to $G^{\prime}<G^{\prime}$ ' and losses its 359 linearity. As illustrated from Figure 3A, the linear viscoelastic region progressively increased from $\mathrm{C} 2$ to $\mathrm{C} 10$. A large linear viscoelastic region in $\mathrm{C} 10$ revealed that $\mathrm{MFC}$ gel was stiff and have a better stability. A larger linear viscoelastic region is preferred when MFC is used as a stabilizer for oil-in-water emulsion since it will provide better stability and greater coating/thickness to the emulsion.

364 As for the frequency sweep test, it can be seen that the frequency has only slight 365 influence on the G' G', for all the samples. An unstable sample will tend to sediment when G' $>$ G'. All the samples exhibited $G$ ' $>$ G' at low and high frequency regions indicating that the MFC was resistant towards long term instability. 


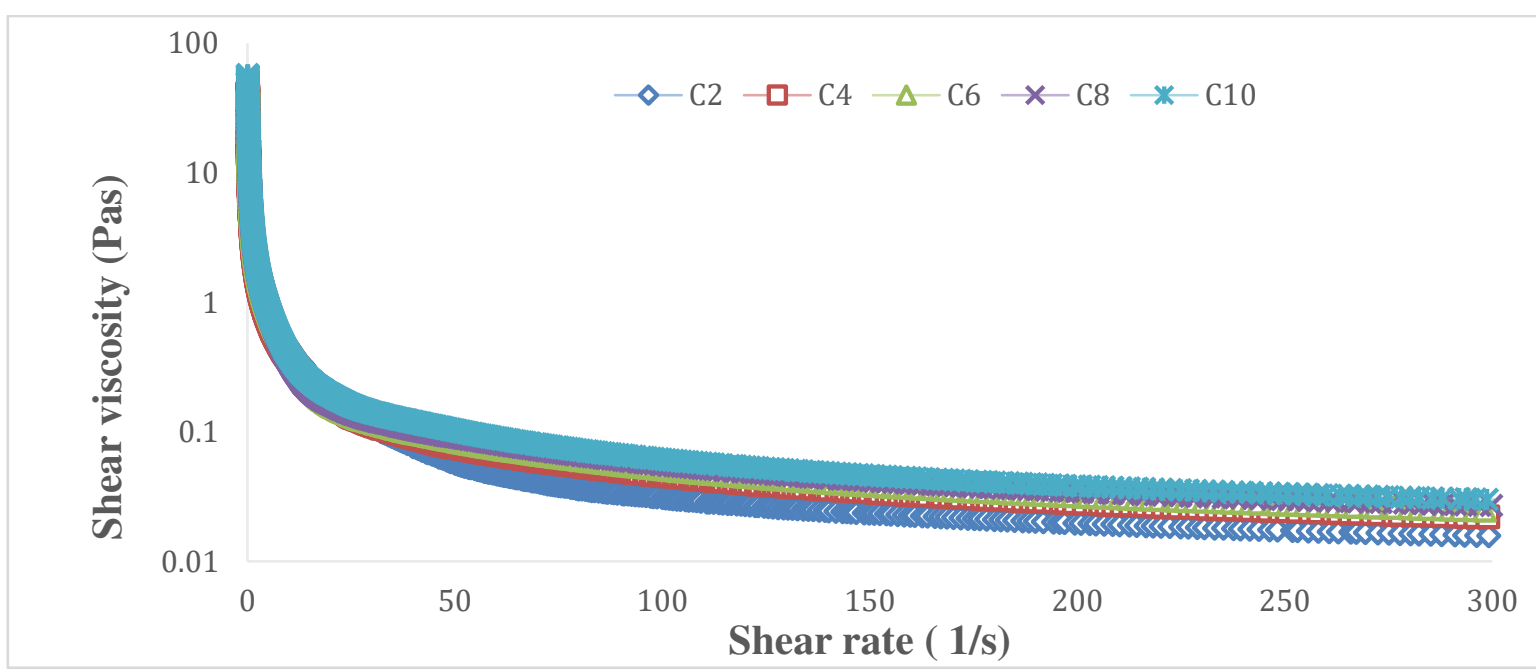

$370 \quad$ B

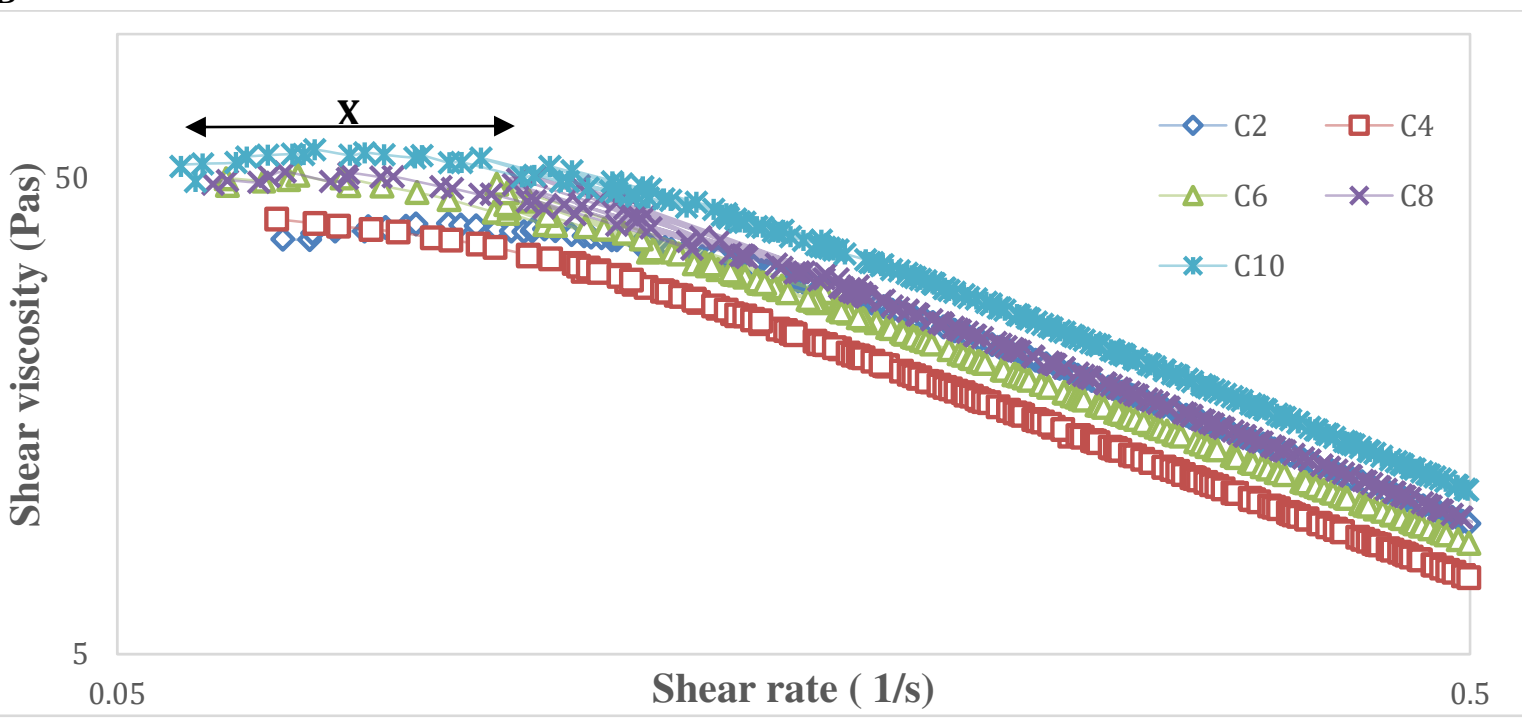

$\mathrm{C}$

374 Figure 2: Flow properties of MFC from different cycles of HPH. (A) shear stress as a function of shear 375 rate, (B) shear stress as a function of shear rate log scale (C) Shear stress as function of shear rate. C0 376 represented cycle 0, C2 represented cycle 2, C4 represented cycle 4, C6 represented cycle 6, C8 represented cycle $8, \mathrm{C} 10$ represented cycle 10 
A

$381 \quad$ B
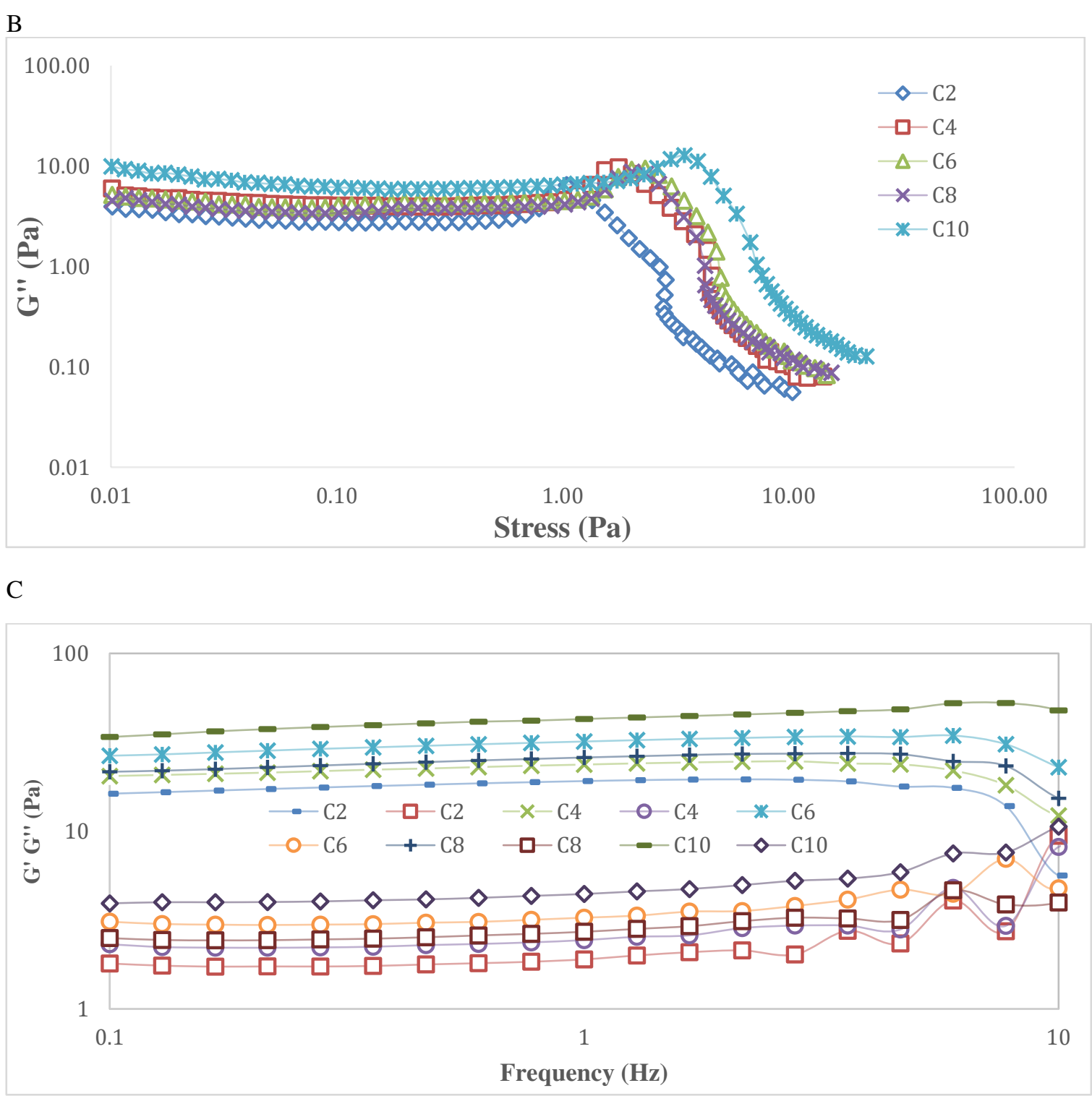

385 Figure 3: Viscoelastic properties of MFC produced from different cycles of HPH (A) storage modulus 386 G' as a function of stress, (B) loss modulus as a function of stress log scale (C) G' (closed symbol) G', 
(open symbol) as function of frequency. $\mathrm{C} 0$ represented cycle $0, \mathrm{C} 2$ represented cycle 2, $\mathrm{C} 4$ represented

\subsection{Emulsion stabilized by MFC prepared from homogenization cycle 0 to cycle 10}

391 MFC produced from cycle 0 to cycle 10 was further utilized to stabilize oil-in-water 392 emulsion and the stability was assessed.

\subsubsection{Particle size}

Table 3 shows the particle size distribution of emulsion stabilized by MFC produced from cycle 0 to cycle 10 . The MFC-stabilized emulsion showed a nearly monomodal particle size distribution (Figure 4). Emulsion stabilized by MFC produced from high pressure homogenizer had mean volume weighted diameter $\left(d_{4,3}\right)$, surface weighted diameter $\left(\mathrm{D}_{3,2}\right), \mathrm{D} 10, \mathrm{D} 50$ and $\mathrm{D} 90$ value that were significantly $(\mathrm{P}<0.05)$ lower than emulsion prepared from MFC without undergoing homogenization. Homogenization resulted in more MFC to be released from the fiber bundle. Furthermore, homogenization also reduced the size of the MFC. Similar result was also disclosed by Winuprasith et al. (2015) who reported that particle size of MFC-stabilized emulsion reduced when MFC of mangosteen rind from higher cycle of homogenization was used to stabilize the emulsion.

Table 3: Particle size profiles of emulsions produced using MFC from different high pressure homogenization cycles.

\begin{tabular}{lllllll}
\hline & \multicolumn{5}{c}{ Droplet mean diameter } \\
\cline { 2 - 6 } HPH cycle & Span index & $d_{4,3}(\mu \mathrm{m})$ & $d_{3,2}(\mu \mathrm{m})$ & $\mathrm{D}(0.1)$ & $\mathrm{D}(0.5)$ & $\mathrm{D}(0.9)$ \\
\hline $\mathrm{C} 0$ & $1.86 \pm 0.03^{\mathrm{a}}$ & $93.20 \pm 10.12^{\mathrm{a}}$ & $17.61 \pm 1.68^{\mathrm{a}}$ & $24.34 \pm 3.09^{\mathrm{a}}$ & $81.78 \pm 7.85^{\mathrm{a}}$ & $176.66 \pm 20.42^{\mathrm{a}}$ \\
$\mathrm{C} 2$ & $1.88 \pm 0.01^{\mathrm{a}}$ & $77.09 \pm 7.93^{\mathrm{b}}$ & $14.59 \pm 1.31^{\mathrm{be}}$ & $17.27 \pm 2.78^{\mathrm{bc}}$ & $68.64 \pm 6.38^{\mathrm{b}}$ & $146.35 \pm 15.66^{\mathrm{b}}$ \\
$\mathrm{C} 4$ & $1.80 \mathrm{v} 0.03^{\mathrm{b}}$ & $76.89 \pm 8.50^{\mathrm{b}}$ & $14.74 \pm 1.51^{\mathrm{be}}$ & $19.39 \pm 2.99^{\mathrm{c}}$ & $69.11 \pm 6.64^{\mathrm{b}}$ & $143.84 \pm 16.94^{\mathrm{b}}$ \\
$\mathrm{C} 6$ & $1.93 \pm 0.02^{\mathrm{c}}$ & $73.65 \pm 7.96^{\mathrm{b}}$ & $13.50 \pm 1.37^{\mathrm{bc}}$ & $14.00 \pm 2.19^{\mathrm{bd}}$ & $66.01 \pm 6.34^{\mathrm{b}}$ & $141.70 \pm 15.92^{\mathrm{b}}$ \\
$\mathrm{C} 8$ & $2.02 \pm 0.01^{\mathrm{d}}$ & $68.68 \pm 5.10^{\mathrm{b}}$ & $11.81 \pm 0.77^{\mathrm{c}}$ & $10.30 \pm 1.41^{\mathrm{d}}$ & $61.34 \pm 3.98^{\mathrm{b}}$ & $134.31 \pm 10.29^{\mathrm{b}}$ \\
$\mathrm{C} 10$ & $1.95 \pm 0.03^{\mathrm{c}}$ & $70.17 \pm 4.88^{\mathrm{b}}$ & $12.54 \pm 0.72^{\mathrm{ce}}$ & $12.70 \pm 1.67^{\mathrm{d}}$ & $62.63 \pm 3.54^{\mathrm{b}}$ & $135.04 \pm 10.27^{\mathrm{b}}$ \\
\hline The C0 represented emulsion produced use MFC from homogenization cycle 0, so on and so forth. The \\
MFC amount in the emulsion were 0.45\%. Test were performed in triplicates. Mean value \pm standard \\
deviation followed by same letter in each column are not significantly different $(\mathrm{P}>0.05)($ span index; \\
$d_{4,3}$ :volume weighted mean diameter; $d 3,2:$ surface weighted mean diameter; D $(0.1), \mathrm{D}(0.5)$ and D $(0.9)$ \\
cumulative distribution of particle size at 10\%, 50\% and 90\%, respectively
\end{tabular}




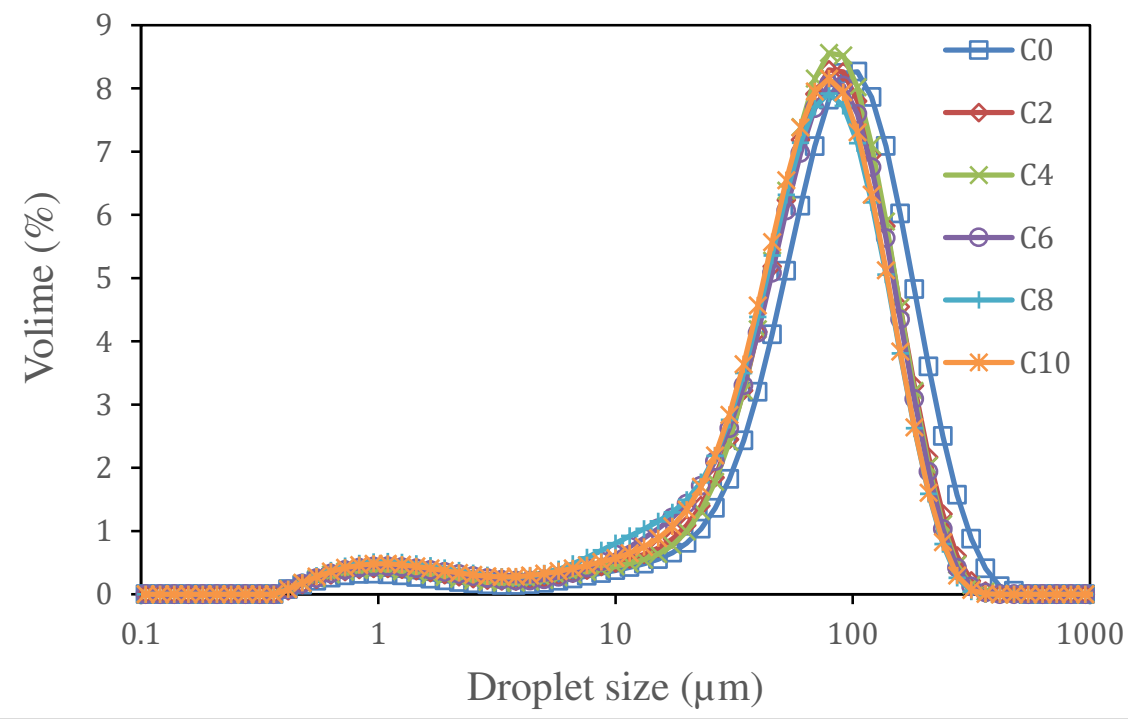

413 Figure 4: Particle size distributions of oil-in-water emulsions stabilized by microfibrillated celluloses 414 prepared from different cycles of homogenizer. (C0 represented cycle $0, \mathrm{C} 2$ represented cycle $2, \mathrm{C} 4$ 415 represented cycle 4, C6 represented cycle 6, C8 represented cycle 8, C10 represented cycle 10)

\section{$417 \quad$ 3.3.2 Accelerated stability}

418 Table 4 shows the accelerated stability of the emulsion stabilized by MFC prepared from 419 cycle 0 to cycle 10 under three different temperatures whereas Figure 5 illustrates the 420 Lumifuge transmission profile of emulsion. CI reduced with the increase in high pressure 421 homogenization cycle. MFC produced at cycle 10 was the most stable where no oiling 422 off was observed showing all emulsions were capable of holding $10 \%$ of oil. All 423 emulsions stored under $5{ }^{\circ} \mathrm{C}$ had a lower $\mathrm{CI}$ as compared to $25^{\circ} \mathrm{C}$ and $45^{\circ} \mathrm{C}$.

424 Table 4: Accelerated creaming index of emulsions prepared from MFC produced by different high 425 pressure homogenization cycles at different temperatures.

\begin{tabular}{cccc}
\hline Homogenization cycle & \multicolumn{3}{c}{ Creaming index $(\%)$} \\
\hline & 5 & 25 & 45 \\
\cline { 2 - 4 } & $40.76 \pm 3.92^{\mathrm{a}^{*}}$ & $39.70 \pm 2.78^{\mathrm{a}^{*}}$ & $44.85 \pm 3.03^{\mathrm{a}^{\prime}}$ \\
\hline 0 & $36.97 \pm 2.33^{\mathrm{b}^{*}}$ & $36.52 \pm 1.84^{\mathrm{b}^{*}}$ & $42.42 \pm 1.84^{\mathrm{a}^{\prime}}$ \\
2 & $32.27 \pm 0.79^{\mathrm{c}^{*}}$ & $32.73 \pm 1.14^{\mathrm{c \textrm {d } ^ { * }}}$ & $35.61 \pm 1.31^{\mathrm{b}}$ \\
4 & $30.45 \pm 1.57^{\mathrm{cd}^{*}}$ & $35.15 \pm 0.52^{\mathrm{bc}}$ & $37.27 \pm 1.64^{\mathrm{b}}$ \\
8 & $27.73 \pm 1.20^{\mathrm{d}^{*}}$ & $33.94 \pm 1.14^{\mathrm{bc}}$ & $35.15 \pm 0.69^{\mathrm{b}}$ \\
10 & $24.55 \pm 1.20^{\mathrm{e}^{*}}$ & $30.76 \pm 0.69^{\mathrm{d}^{\prime}}$ & $31.82 \pm 0.45^{\mathrm{c}^{\prime}}$
\end{tabular}
were performed in triplicates. Mean value \pm standard deviation followed by same letter in each column are not significantly different $(\mathrm{P}>0.05)$. For pairwise comparison of different temperatures for each individual HPH cycle the same symbol $\left({ }^{*}\right)$ in each row are not significantly different $(\mathrm{P}>0.05)$. 


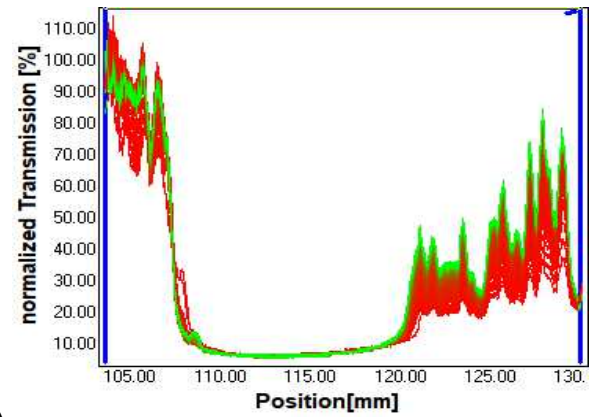

A
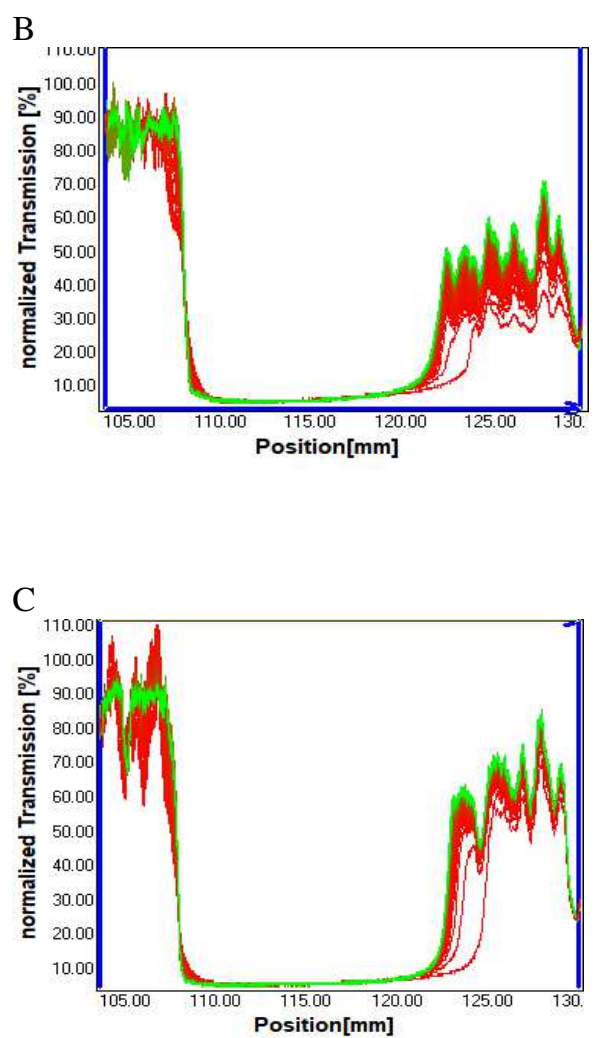

$\mathrm{D}$

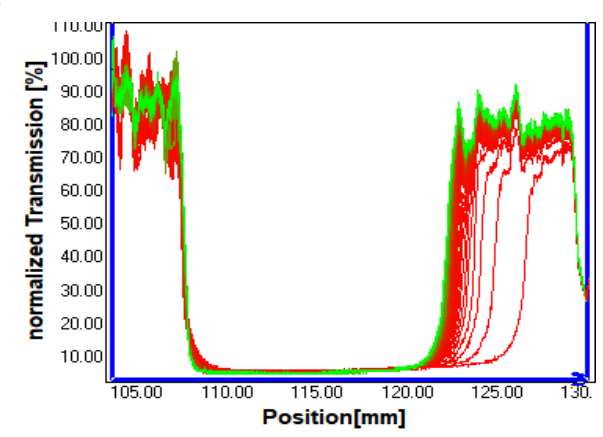

$\mathrm{E}$

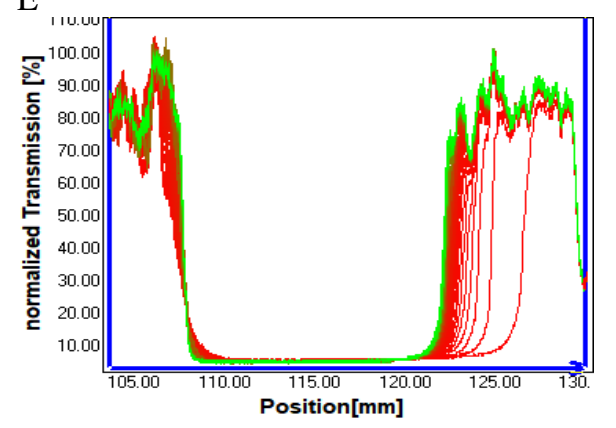

$\mathrm{F}$

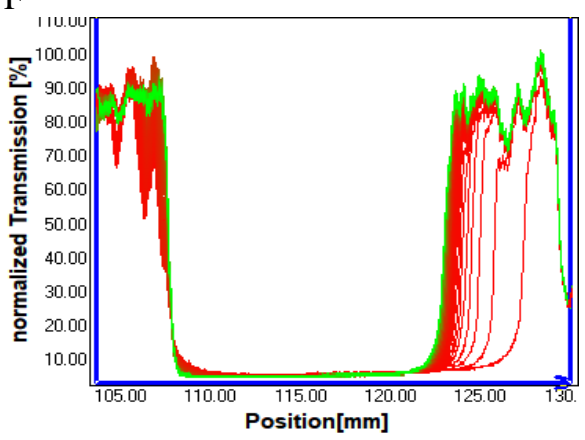
cycles of high pressure homogenization at $25^{\circ} \mathrm{C}$.

435 (A) cycle 0, (B) cycle 2, (C) cycle 4 (D) cycle 6 (E) cycle 8 (F) cycle 10. Photograph of emulsion using MFC cycle 4 and cycle 10 at $25^{\circ} \mathrm{C}$. 


\section{$439 \quad$ 3.3.3.1 Flow behavior}

440 Figure 6 A shows the steady flow behavior of emulsions stabilized by MFC produced 441 from cycle 0 to cycle 10. All emulsions prepared from MFC showed shear thinning 442 (pseudoplastic) behavior. Similar observation was found in many emulsion systems that 443 is stabilized by polysaccharides such as chitosan, gum Arabic and microcrystalline 444 cellulose (Burr et al. 2018; Jia et al. 2015). However, the shear thinning effect only 445 reduced slightly when with the cycles of homogenization. This may be due to more MFC 446 being released under high HPH cycles that creates a stronger fiber network causing the 447 emulsion to be more resistant to shear.

448 Zero shear viscosity is viscosity of sample when shear approaches zero (no shear) that 449 demonstrates the viscosity of sample at stand still condition when no force is being 450 applied. A higher zero shear viscosity value is important in regards to the product 451 stability. An emulsion with higher zero shear viscosity will have higher stability as it 452 restricted the movement of molecules and reduced their overall kinetic energy. Therefore, 453 lesser molecules can overcome the energy barrier to coalesce. The emulsions produced 454 using MFC from cycles 0 to 10 had zero shear viscosity around 3 Pa.s (Figure 5 A). It 455 implies that the emulsion system may have similar stability. Nevertheless, MFC 456 produced from higher cycles performed better in stabilizing emulsion since its overall 457 shear viscosity is the highest.

458 The degrees of thixotropy of emulsions produced were tabulated in Table 5. A sample is 459 called thixotropy (time-dependent shear thinning property) when it is tested with 460 ascending shear rate followed by descending shear rate and create a hysteresis loop. The 461 area of the hysteresis loop is the energy consumed for structural breakdown. Despite 462 significant difference was observed between $\mathrm{C} 2$ to $\mathrm{C} 10$ emulsions $(\mathrm{P}>0.05)$, thixotropy 463 of the emulsion gradually increased from 350 to around 450 with exception of emulsions 464 C6. Emulsions made up of more MFC like C8 and C10 is believed to possess more 465 complex network structure that required longer time for structure restoration.

\section{3.3.3.2 Phase angle and G'G'” crossover}

467 Figure $6 \mathrm{~B}$ illustrates the phase angle distributions of emulsions stabilized by MFC 468 produced from cycle 0 to cycle 10 . Phase angle provide information related to whether 
sample deform elastically like a solid in low phase angle $\left(0^{\circ}\right)$ or flow like a liquid at high

470 phase angle $\left(90^{\circ}\right)$. All emulsions had a plateau phase angle around $8^{\circ}$ showing that the

471 emulsions have intact elastic-like structure at low stress (amplitude). However, when the 472 stress forces increased, the phase angle shifted to the region that belongs to liquid 473 dominant $\left(90^{\circ}\right)$.

474 Table 5 shows the as single point yield stress. A yield is when a certain force is applied 475 to a certain extent that exceeded a threshold that caused a gel or viscoelastic solid start to 476 flow. It should be noted that yield is a process. The transition of solid to liquid occur 477 (G'G', crossover) is interpolated by the phase angle $45^{\circ}$ (Shih et al. 1999). The crossover 478 occurred at a higher Pa corresponding to MFC produced at $\mathrm{C} 10$ which is $7.83 \mathrm{~Pa}$. Under 479 high HPH cycles, more MFC are released in the fiber suspension to create a strong 3D 480 network for supporting the emulsion system. Therefore, a high yield stress (force) is 481 required to disrupt the network in order to collapse the emulsion system allowing it to 482 flow like a liquid. This property is applicable in many products like mayonnaise which 483 stand still like a solid when no external forces were applied, but starting to flow like a 484 liquid when pressure was applied. Hence, yield stress for a product should be conditional 485 not absolute.

486 As for the frequency sweep test, the storage modulus $G^{\prime}$ for all emulsions were always 487 higher than loss modulus G', independent to the frequency (Figure 6 C-D). It showed 488 that the emulsions possesses gel-like behavior. The magnitude of G'G', increased with 489 HPH cycles demonstrating that the gelation properties were greatly influenced by the 490 HPH cycle. Emulsion with a greater gelation properties can be obtained if MFC from 491 higher HPH cycles were used. 


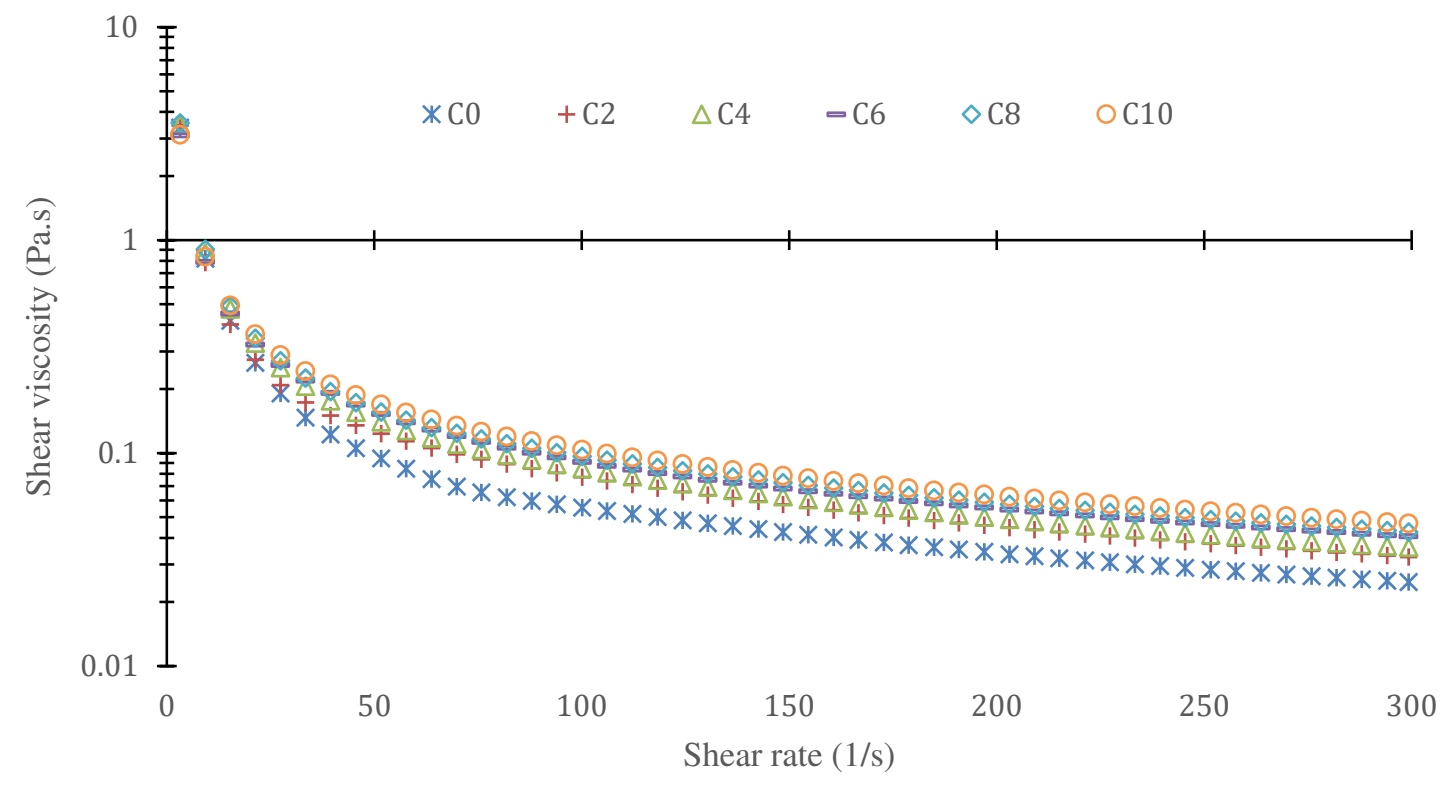

500

501

502

503

B

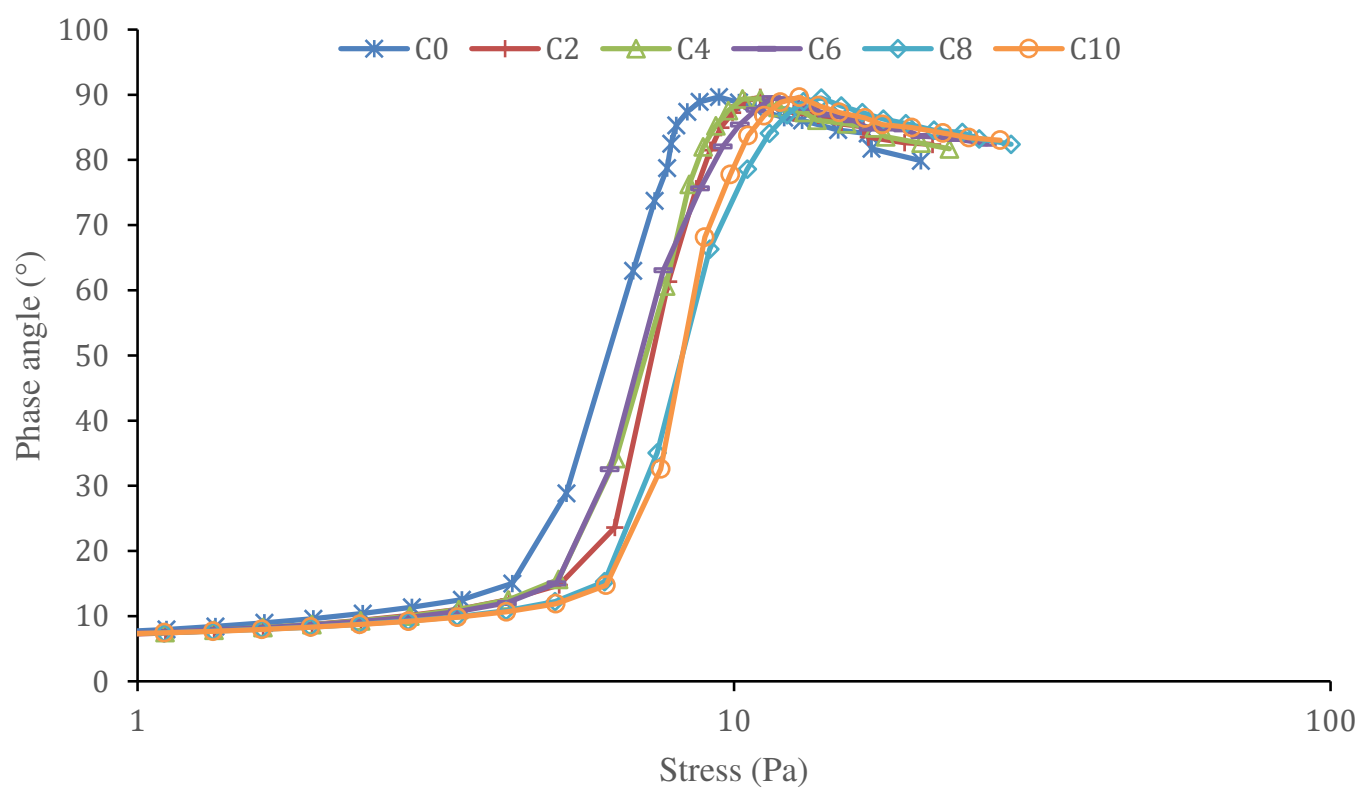

504

505

506

507

508

509

510

511

512

513

514

515 

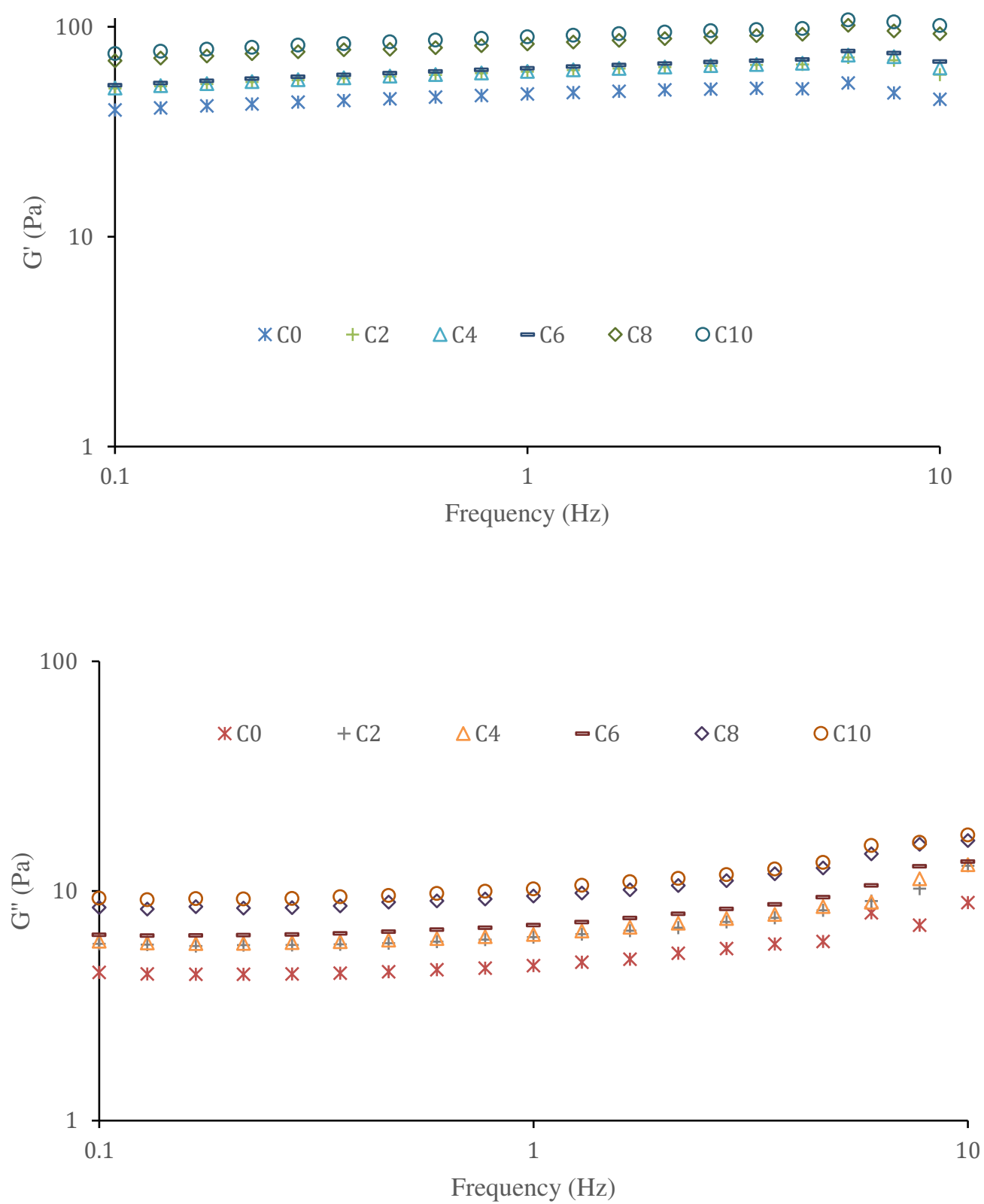

Figure 6: Rheological behavior of emulsions produced using different cycles of microfibrillated cellulose. (A) shear stress as a function of shear rate, (B) phase angle as a function shear stress (C) storage modulus G' and (D) loss modulus G' as a function of angular frequency measured at temperature of $25^{\circ} \mathrm{C}$.

525 The C0 represented emulsion produced using MFC from homogenization cycle 0 , so on and so forth. The final MFC amount in the emulsions were $0.45 \%$.

Table 5: Thixotropy and G'G', crossover of emulsion stabilized by using MFC produced from different cycles of high pressure homogenization

\begin{tabular}{|c|c|c|c|}
\hline $\begin{array}{l}\text { Homogenization } \\
\text { of MFC }\end{array}$ & cycle & Hysteresis area $(\mathrm{Pa} / \mathrm{s})$ & $\mathrm{G}^{\prime} \mathrm{G}^{\prime \prime}$ crossover $(\mathrm{Pa})$ \\
\hline $\mathrm{CO}$ & & $350.47 \pm 13.45^{\mathrm{a}}$ & $6.03 \pm 0.26^{\mathrm{a}}$ \\
\hline $\mathrm{C} 2$ & & $424.87 \pm 26.70^{\mathrm{b}}$ & $7.15 \pm 0.22^{\mathrm{bc}}$ \\
\hline
\end{tabular}




\begin{tabular}{lll}
\hline C4 & $453.30 \pm 7.46^{\mathrm{b}}$ & $6.91 \pm 0.79^{\mathrm{ab}}$ \\
C6 & $420.77 \pm 45.42 \mathrm{~b}$ & $6.70 \pm 0.41^{\mathrm{ab}}$ \\
C8 & $477.93 \pm 44.63^{\mathrm{b}}$ & $7.85 \pm 0.47^{\mathrm{c}}$ \\
$\mathrm{C} 10$ & $465.63 \pm 54.90^{\mathrm{b}}$ & $7.83 \pm 0.66^{\mathrm{c}}$ \\
\hline
\end{tabular}

530 The $\mathrm{C} 0$ represented emulsion produced using MFC from homogenization cycle 0, so on and so forth. The 531 final MFC amount in the emulsion were $0.45 \%$. Test were performed in triplicates. Mean 532 value \pm standard deviation followed by same letter in each column are not significantly different $(\mathrm{P}>0.05)$

\subsection{Emulsion stabilized by 0\%-1\% of MFC}

As MFC produced after undergoing 10 cycle of homogenization was the most stable, it was in different amount from $0 \%$ to $1 \%$ to assess the amount of MFC on emulsion stability.

\subsubsection{Particle size}

Table 6 shows the of particle size distribution of emulsion stabilized by $0 \%$ to $1 \%$ of MFC. Volume weighted diameter $d_{4,3}$ of the emulsion significantly increased $(\mathrm{P}<0.05)$ with MFC amount. In general, emulsion with smaller particles had higher stability.

542 However, it should be noted that this theory only applies to spherical particle. Hence, 543 MFC-stabilized emulsions might be different since MFC is usually in elongated rod 544 shape in structure. The bimodal model distribution observed in present study showed the 545 possibility that small particle size $(\sim 1 \mu \mathrm{m})$ might be contributed by the oil droplets that is not trapped by MFC while a bigger particle size may be attributed to the emulsion where the oil was entrapped in the three dimensional network of MFC (Figure 7). Hence, it proved that a stable MFC-stabilized emulsion will have a larger particle size $d_{4,3}$.

Table 6: Particle size profiles of emulsions produced using MFC with different final amounts of MFC.

\begin{tabular}{lllllll}
\hline & \multicolumn{5}{c}{ Droplet mean diameter } \\
\cline { 2 - 6 } Amoun (\%) & Span index & $d_{4,3}(\mu \mathrm{m})$ & $d_{3,2}(\mu \mathrm{m})$ & $\mathrm{D}(0.1)$ & $\mathrm{D}(0.5)$ & $\mathrm{D}(0.9)$ \\
\hline 0.00 & $2.14 \pm 0.04^{\mathrm{a}}$ & $3.05 \pm 0.05^{\mathrm{a}}$ & $1.74 \pm 0.00^{\mathrm{a}}$ & $0.91 \pm 0.00^{\mathrm{a}}$ & $2.05 \pm 0.01^{\mathrm{a}}$ & $5.29 \pm 0.07^{\mathrm{a}}$ \\
0.25 & $2.50 \pm 0.02^{\mathrm{b}}$ & $27.18 \pm 0.81^{\mathrm{b}}$ & $6.09 \pm 0.11^{\mathrm{b}}$ & $1.86 \pm 0.04^{\mathrm{a}}$ & $22.52 \pm 0.57^{\mathrm{b}}$ & $58.15 \pm 1.83^{\mathrm{b}}$ \\
0.50 & $1.91 \pm 0.03^{\mathrm{b}}$ & $71.50 \pm 4.14^{\mathrm{c}}$ & $13.15 \pm 0.59^{\mathrm{c}}$ & $13.25 \pm 1.04^{\mathrm{b}}$ & $64.53 \pm 3.04^{\mathrm{c}}$ & $136.61 \pm 8.57^{\mathrm{c}}$ \\
0.75 & $2.03 \pm 0.03^{\mathrm{c}}$ & $73.96 \pm 3.01^{\mathrm{cd}}$ & $12.48 \pm 0.32^{\mathrm{d}}$ & $10.84 \pm 0.52^{\mathrm{b}}$ & $65.54 \pm 2.03^{\mathrm{cd}}$ & $143.77 \pm 6.66^{\mathrm{cd}}$ \\
1.00 & $1.98 \pm 0.03^{\mathrm{c}}$ & $77.81 \pm 2.71^{\mathrm{d}}$ & $14.21 \pm 0.16^{\mathrm{e}}$ & $15.48 \pm 0.32^{\mathrm{b}}$ & $68.55 \pm 1.87^{\mathrm{d}}$ & $150.96 \pm 6.43^{\mathrm{d}}$ \\
\hline MFC used were from high pressure homogenization cycle 10. Test were performed in triplicates. Mean \\
value \pm standard deviation followed by same letter in each column are not significantly different $(\mathrm{P}>0.05)$. \\
(span index; $d_{4,3}$ : volume weighted mean diameter; $d_{3,2}:$ surface weighted mean diameter; D $(0.1), \mathrm{D}(0.5)$ \\
and D $(0.9)$ cumulative distribution of particle size at 10\%, 50\% and 90\%, respectively.
\end{tabular}




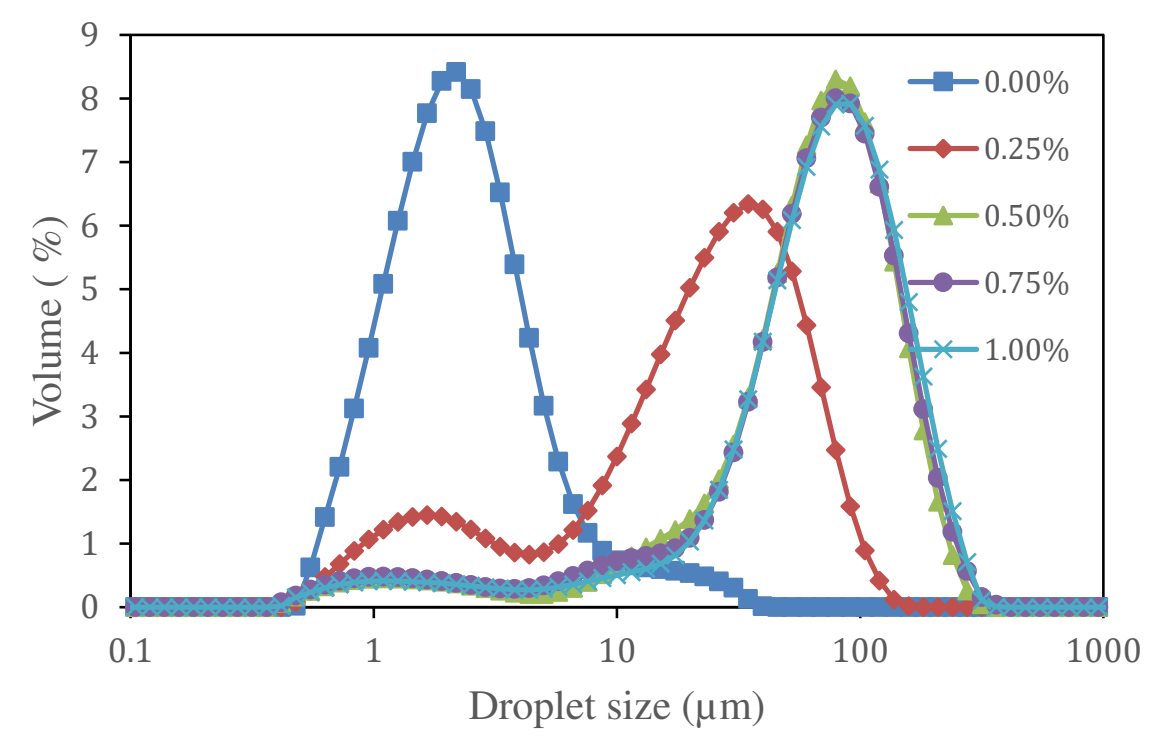

557 Figure 7: Particle size distributions of oil-in-water emulsions stabilized by $0 \%, 0.25 \%, 0.50 \%, 0,75 \%$, $558 \quad \mathbf{1 . 0 0} \%$ amount of microfibrillated celluloses. The final MFC amount in the emulsions were $0.45 \%$ in the 559 cycle study. MFC used in the amount study were from high pressure homogenization cycle 10.

\section{$560 \quad 3.4 .2$ Accelerated stability}

561 Table 7 shows the accelerated stability of the emulsions stabilized by $0 \%$ to $1 \%$ of (w/w) 562 of MFC under different thermal environments. Figure 8 shows the lumifuge profile of 563 the emulsion. Emulsion produced tended to have a high stability and lower creaming 564 behavior when high amount of MFC was used. For instance, CI of MFC emulsions 565 reduced from $57.42 \%$ to $2.73 \%$ when MFC amount increased from $0.25 \%$ to $1.0 \%$. A 566 high amount of MFC was postulated to restrict the movement of oil droplets thereby

Table 7: Accelerated Creaming index of emulsions prepared by different amount MFC at different temperatures.

\begin{tabular}{cccc}
\hline MFC amount (w/w) \% & \multicolumn{3}{c}{ Creaming index (\%) } \\
\cline { 2 - 4 } & \multicolumn{3}{c}{ Temperature $\left({ }^{\circ} \mathrm{C}\right)$} \\
\cline { 2 - 4 } & 5 & 25 & 45 \\
\hline 0.00 & $21.67 \pm 0.95$ & $38.18 \pm 0.45$ & $55.45 \pm 0.45$ \\
0.25 & $42.12 \pm 0.95^{\mathrm{a}^{*}}$ & $57.42 \pm 0.95^{\mathrm{a}^{\prime}}$ & $54.39 \pm 0.69^{\mathrm{a}^{\prime}}$ \\
0.50 & $24.85 \pm 0.69^{\mathrm{b}^{*}}$ & $31.21 \pm 3.47^{\mathrm{b}^{\prime}}$ & $33.18 \pm 1.36^{\mathrm{b}^{\prime}}$ \\
0.75 & $4.70 \pm 1.14^{\mathrm{c}^{*}}$ & $12.73 \pm 1.20^{\mathrm{c}^{\prime}}$ & $15.15 \pm 1.60^{\mathrm{c}^{\prime}}$ \\
1.00 & $4.55 \pm 1.64^{\mathrm{c}^{*}}$ & $2.73 \pm 0.45^{\mathrm{d}^{*}}$ & $3.03 \pm 0.26^{\mathrm{d}^{*}}$ \\
\hline
\end{tabular}

570 MFC used were from high pressure homogenization cycle 10. Tests were performed in triplicates. Mean 571 value \pm standard deviation followed by same letter in each column are not significantly different $(\mathrm{P}>0.05)$. 572 For pairwise comparison of different temperatures for each individual HPH cycle the same symbol (*') in 573 each row are not significantly different $(\mathrm{P}>0.05)$. 
A
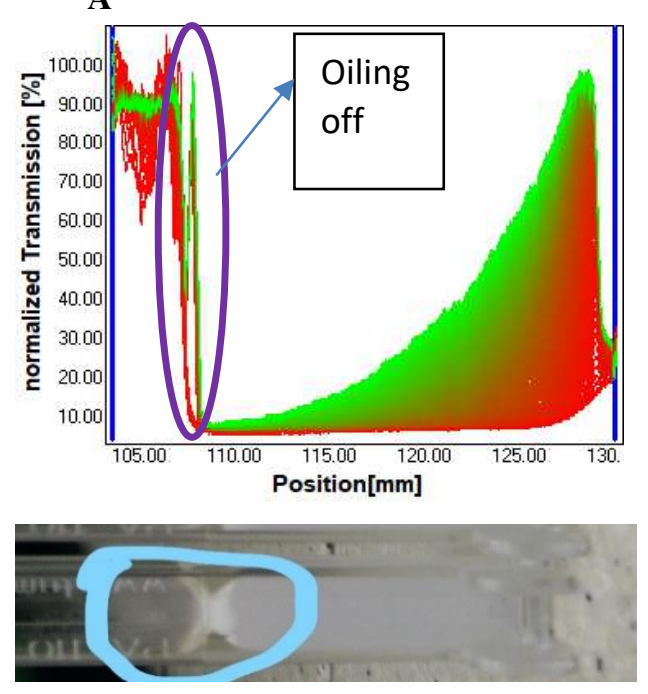

B
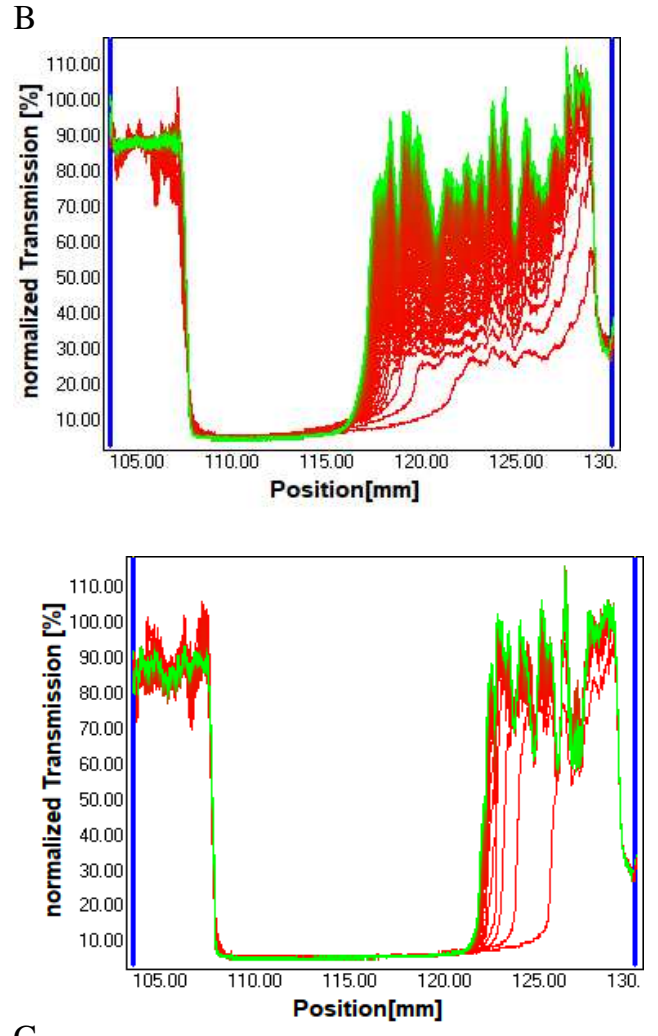

$\mathrm{D}$
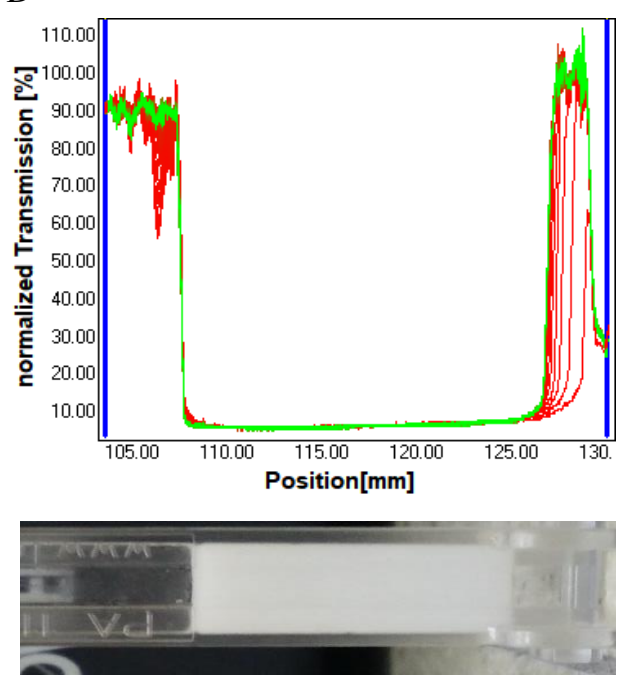

$\mathrm{E}$
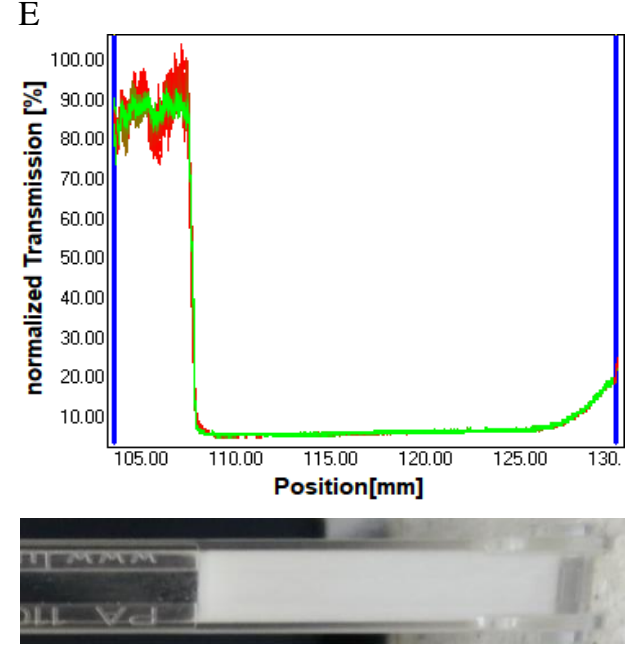

Figure 8: Lumifuge transmission profile over time for emulsions stabilized prepared with different 576 final amounts of $\mathrm{MFC}$ at $25^{\circ} \mathrm{C}$.

577 (A) $0.00 \%$, (B) $0.25 \%$, (C) $0.5 \%$ (D) $0.75 \%$ and (E) $1.00 \%$ MFC with (A), (D) and (E) having photo of 578 emulsions containing tubes with its respective final amount of MFC.

\section{$579 \quad$ 3.4.3 Rheology}

\subsubsection{Flow behavior}

581 Figure 9 A shows the steady flow curve of emulsions stabilized by $0 \%-1 \%$ of MFC.

582 Emulsion stabilized by $0.25 \%$ MFC possessed the lowest shear viscosity. Shear viscosity increased with the amount of MFC up to $0.75 \%$ and plateau at $1 \%$ of MFC. A slight 
584 increase in MFC amount increase the apparent viscosity drastically. It was found that the 585 shear thinning behavior was more pronounced at higher concentrations of MFC. A high 586 MFC concentration creates a compact flocculated droplets entrapped by MFC network. 587 Hence, the flocs became more sensitive to shear force when exists in a closed packed 588 arrangement. When shear force is exerted, it causes rearrangement of shape, 589 disintegration of flocs and disruption of MFC networks. As a result, the droplets moved 590 in a parallel position causing the viscosity to reduce (Quemada and Berli 2002). It should 591 be noted that the reduction of shear viscosity for $1.00 \%$ MFC stabilized-emulsion was 592 from $\sim 100$ at 0 Pa.s to $\sim 0.1$ Pa.s at $300 \mathrm{~s}^{-1}$ shear rate. However, 1.00\% of MFC stabilized 593 emulsion still possesses higher shear viscosity as compared to other emulsions.

594 The thixotropy of emulsions stabilized by $0 \%$ to $1 \%$ of MFC is tabulated in Table 8 . The 595 result showed that thixotropy value in emulsion significantly $(\mathrm{p}<0.05)$ increased from $59690.89(0.25 \%$ MFC) to 2657 (1.00\% MFC) with a slight increase in the MFC amount. 597 Similar property was also exhibited in many polysaccharide stabilizers due to the 598 presence of large amounts of available binding sites for hydrogen bonding. A slight 599 increase in MFC amount can create more entanglement and binding region between the 600 molecules which further promote the development of a strong network. Hence, it takes a 601 longer time for the structure to recover thereby creating a larger thixotropy areas.

\section{3.4.3.2 Phase angle and G'G', crossover}

604 The phase angle distribution of the emulsion stabilized by $0 \%-1 \%$ of MFC is shown in 605 Figure $9 \mathrm{~B}$. When the amount of MFC increased from $0.25 \%$ to $1.00 \%$, the phase angles 606 plateau area extended significantly $(\mathrm{P}<0.05)$ from $0.01 \mathrm{~Pa}$ to $\sim 10 \mathrm{~Pa}$ (Figure $9 \mathrm{~B}$ ). 607 Emulsion stabilized by $1.00 \%$ of MFC had yield stress increased from 5.65Pa to 28.77 $\mathrm{Pa}$, 608 when MFC concentration increases from $0.5 \%$ to $1.0 \%$. It implies that $1.00 \%$ MFC 609 stabilized emulsion is two times harder to push for it to move/flow then emulsion 610 prepared from $0.5 \%$ MFC. A higher yield stress value also indicates a more stable 611 emulsion. Interestingly, unlike the effect of HPH cycle of MFC on the stability of 612 emulsion, the yield stress were not as significant as compared to amount of MFC. A 613 slight increment in the percentage of MFC was able to provide a strong structural 614 network of MFC to stabilize emulsion due to the extremely high water holding capability 615 of MFC (Siró and Plackett 2010). 
616 Figure 9C-D shows the study of frequency sweep test to assess the viscous-elastic 617 behavior of the material. From Figure $5 \mathrm{C}$, it was observed that emulsion with higher 618 amounts of MFC had a larger G' values. A larger G' value from the frequency sweep test 619 indicates a stronger of MFC network present in the emulsion and therefore less 620 susceptible to deformation without losing its elasticity. For a strong gel, the G' and G', 621 are independent of the test frequency, and the G' was always higher than the G', The 622 emulsion that stabilized by high amount of MFC which was $1.00 \%$ and $0.75 \%$ exhibited 623 a longer frequency independency of G'G" as compared to the emulsion having a lower 624 concentration of MFC. It implies that $1.00 \%$ and $0.75 \%$ of MFC-stabilized emulsion 625 possessed better gelling properties. The emulsion was able to sustain similar magnitude 626 of disturbance (fixed amplitude) for many times $(\mathrm{Hz})$ and still having a good restoration 627 property (G' and G' 'value were not influenced).

628 A

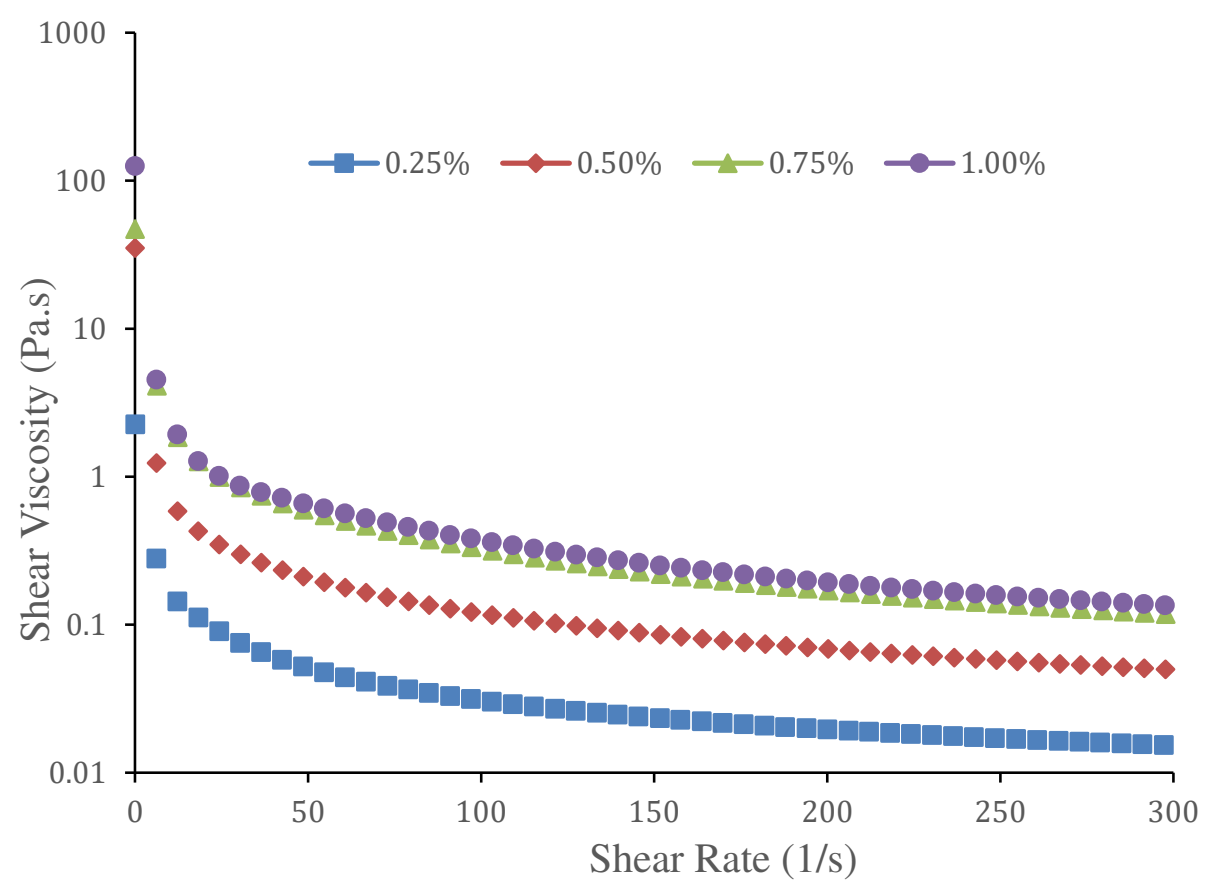




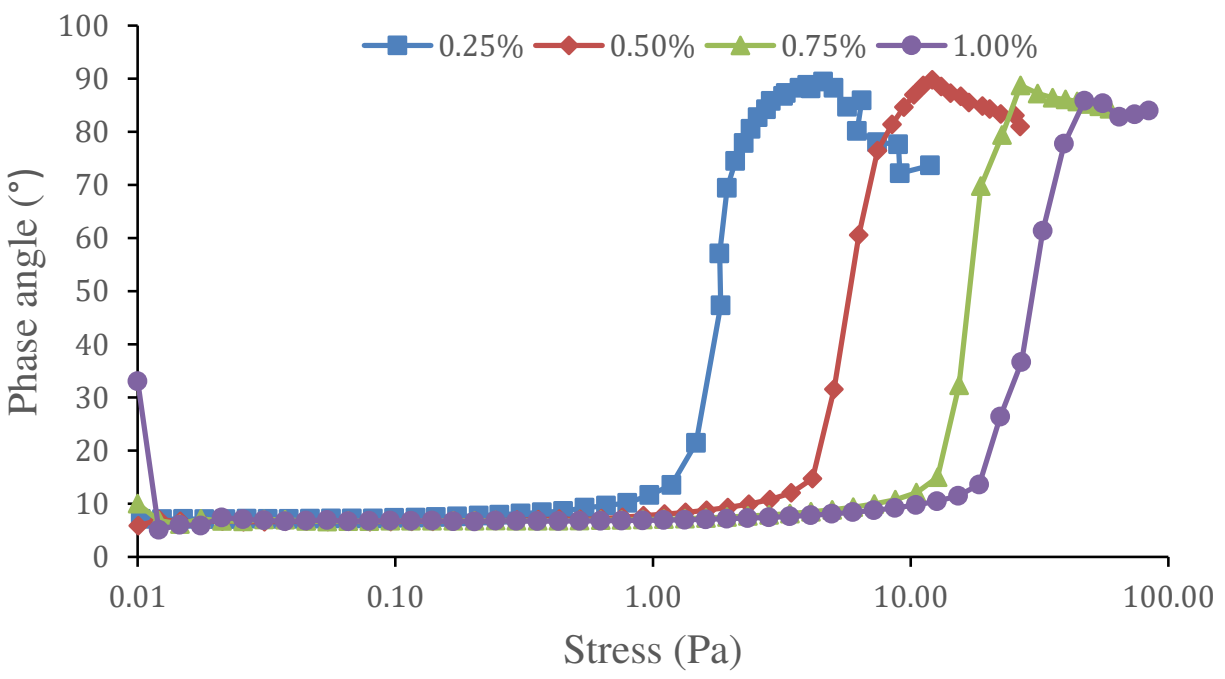

644

C
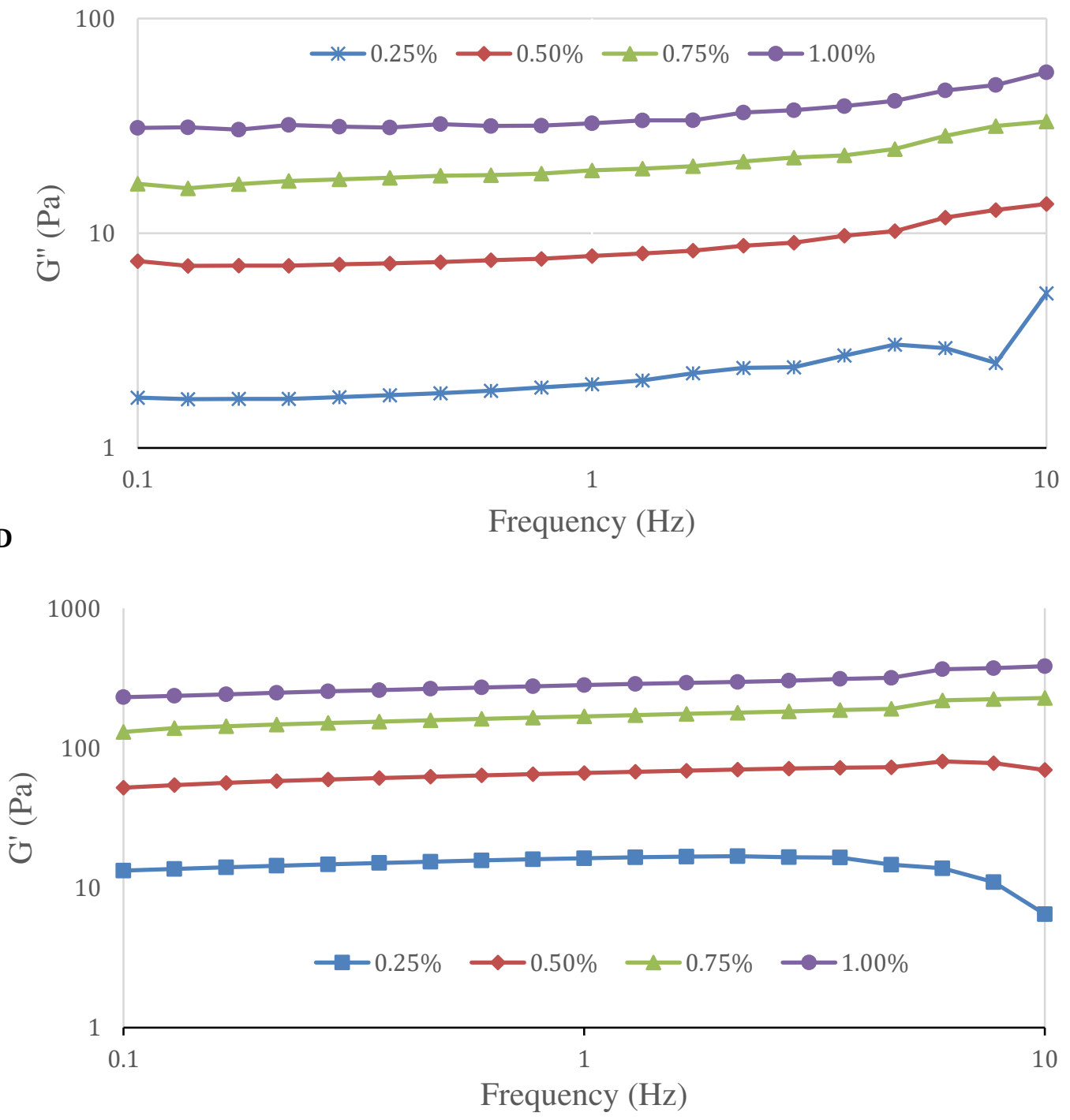

648 Figure 9 Effect of different amounts of microfibrillated cellulose on the rheological behavior of emulsions. 649 The amount of MFC refers to the final amount of MFC in the emulsion and the MFC used were cycle 10. 
(A) shear stress as a function of shear rate, (B) phase angle as a function shear stress (C) storage modulus

Table 8: Thixotropy and G'G', crossover of emulsion stabilized by using different amounts of MFC.

\begin{tabular}{lll}
\hline MFC amount $(\%, w / w)$ & Hysteresis area $(\mathrm{Pa} / \mathrm{s})$ & $\mathrm{G}^{\prime} \mathrm{G}^{\prime \prime}$ crossover $(\mathrm{Pa})$ \\
\hline 0.00 & - & - \\
0.25 & $90.89 \pm 6.19^{\mathrm{a}}$ & $1.87 \pm 0.40^{\mathrm{a}}$ \\
0.50 & $569.60 \pm 26.55^{\mathrm{a}}$ & $5.65 \pm 0.68^{\mathrm{a}}$ \\
0.75 & $2299.67 \pm 54.45^{\mathrm{b}}$ & $16.46 \pm 0.34^{\mathrm{b}}$ \\
1.00 & $2657.00 \pm 550.90^{\mathrm{b}}$ & $28.76 \pm 6.22^{\mathrm{c}}$ \\
\hline
\end{tabular}

654 The amount of MFC refers to the final amount of MFC in the emulsion and the MFC used were cycle 10.

655 Tests were performed in triplicates. Mean value \pm standard deviation followed by same letter in each 656 column are not significantly different $(\mathrm{P}>0.05)$

\subsection{Morphologies of the MFC-stabilized emulsions}

658 Figure 10 illustrates the photomicrographs of MFC-stabilized emulsions prepared using $659 \mathrm{HPH}$ at cycle 2 and cycle 10 viewed under field emission analytical electron microscope 660 (FEEM). The MFC network structure showed to possess thread-like network structure 661 whereas oil droplets was observed as whitish spot with irregular shapes. The images 662 confirmed the entrapment of oil droplet in the MFC network. TEM also revealed that the 663 MFC had diameter in in nanosize range and length that is estimated up to several microns 664 in length.
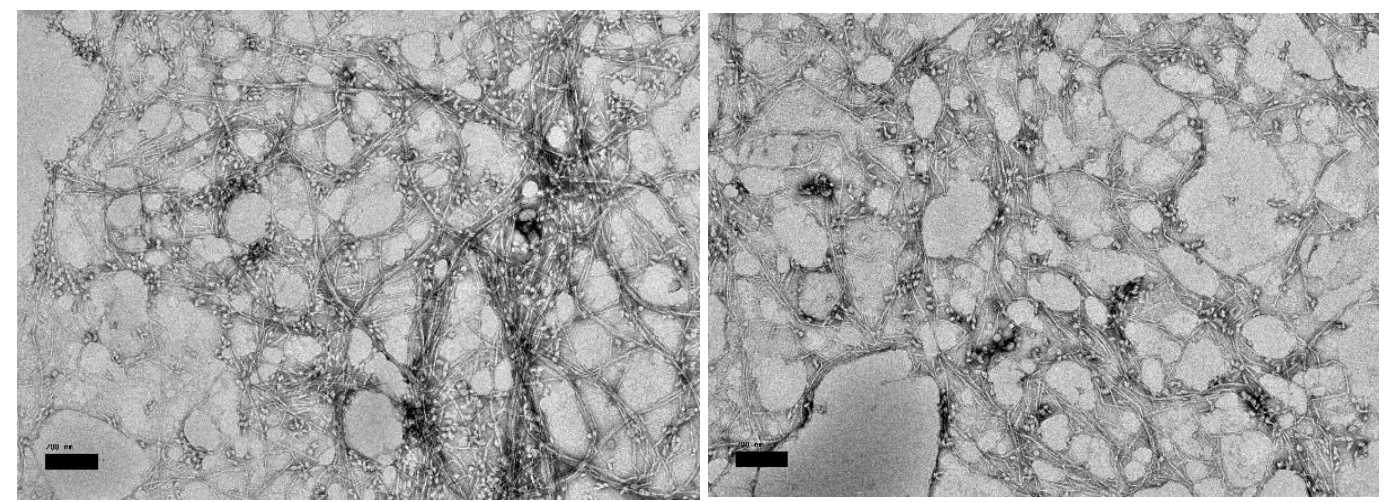

Figure 10: Morphologies of the microfibrillated cellulose-stabilized oil-in-water emulsions prepared using MFC produced from high pressure homogenization cycle 2 (left) and 10 (right) when observed under field emission analytical electron microscope with magnification power of 10000x.

\section{Conclusion}

672 Underutilized corn cob can be converted into MFC using high pressure homogenization 673 aided with endoglucanse Fibercare R enzyme. Addition of $0.02 \%$ of enzyme 
674 endoglucanse is sufficient to facilitate the softening of fiber and prevent blockage of high 675 pressure homogenizer for MFC production. MFC released from the fiber bundle 676 increased with the number of cycles of homogenization. MFC showed to possess gel like 677 consistency with high water holding capacity. MFC was able to stabilize $10 \%$ oil-in678 water emulsion owning to its ability to form three dimensional network structures that 679 can restrict the movement of emulsion droplet. Emulsion stabilized by MFC produced at 680 high cycle of homogenization had a smaller particle sizes and better stability than those 681 produced at low cycle of homogenization. Similarly, a higher amount of MFC resulted in 682 a more stable emulsion even though the emulsion droplet size is bigger due to 683 interconnected fibrous network. All emulsions stabilized by MFC was relatively more 684 stable than those without MFC. The gel like property of the MFC is important in food 685 industry to act as stabilizer for food emulsion. It allows food manufacturers to 686 manipulate the viscosity of the food products particularly for high fat food products such 687 as mayonnaise which commonly need a high percentage of oil to achieve the desirable 688 apparent viscosity. Incorporation of MFC not only can lower the amount of oil used but 689 also and help to maintain viscosity of the products. This approach is important 690 particularly in producing low calorie product that is deemed to be much healthier.

\section{Acknowledgments}

692 This project was funded by Universiti Putra Malaysia under the Putra Grant "Geran 693 Penyelidikan Individu Berprestasi Tinggi” (Project number: GP/IBT/9407400).

695 Ethics Declaration (Conflict of Interest)

696 The authors decale that they have no conflicts of interest. 


\section{References}

Anderson, D., Eastwood, M. (1989). The safety of gum arabic as a food additive and its energy value as an ingredient: a brief review. J Hum Nutr Diet, 2, 137-144.

Burr, S. J., Williams, P. A., Ratcliffe, I. (2018). Synthesis of cationic alkylated chitosans and an investigation of their rheological properties and interaction with anionic surfactant. Carbohydr Polym, 201, 615-623. doi: https://doi.org/10.1016/j.carbpol.2018.08.105

Frelichowska, J., Bolzinger, M.-A., Chevalier, Y. (2010). Effects of solid particle content on properties of o/w Pickering emulsions. J Colloid Interface Sci, 351, 348-356. doi: https://doi.org/10.1016/j.jcis.2010.08.019

Gradinaru, C., Barbuta, M., Babor, D., Serbanoiu, A. (2018). CORN COB ASH AS SUSTAINABLE PUZZOLANIC MATERIAL FOR AN ECOLOGICAL CONCRETE. Bulletin of the Transilvania University of Brasov. Engineering Sciences. Series I, 11, 61-66.

Henriksson, M., Henriksson, G., Berglund, L. A., Lindström, T. (2007). An environmentally friendly method for enzyme-assisted preparation of microfibrillated cellulose (MFC) nanofibers. Eur Polym J, 43, 3434-3441. doi: https://doi.org/10.1016/j.eurpolymj.2007.05.038

Jia, X., Xu, R., Shen, W., Xie, M., Abid, M., Jabbar, S., Wanga, P., Zenga, X., Wu, T. (2015). Stabilizing oil-in-water emulsion with amorphous cellulose. Food Hydrocoll, 43, 275-282. doi: https://doi.org/10.1016/j.foodhyd.2014.05.024

Kalashnikova, I., Bizot, H., Cathala, B., Capron, I. (2011). New Pickering emulsions stabilized by bacterial cellulose nanocrystals. Langmuir, 27, 7471-7479.

Laca, A., Paredes, B., Díaz, M. (2010). A method of egg yolk fractionation. Characterization of fractions. Food Hydrocoll, 24, 434-443. doi: https://doi.org/10.1016/j.foodhyd.2009.11.010

Meena, R., Kesari, K. K., Rani, M., Paulraj, R. (2012). Effects of hydroxyapatite nanoparticles on proliferation and apoptosis of human breast cancer cells (MCF-7). J Nanopart Res, 14, 712.

Pointner, M., Kuttner, P., Obrlik, T., Jäger, A., Kahr, H. (2014). Composition of corncobs as a substrate for fermentation of biofuels. Agron Res, 12, 391-396.

Quemada, D., Berli, C. (2002). Energy of interaction in colloids and its implications in rheological modeling. Adv Colloid and Interface Sci, 98, 51-85. doi: https://doi.org/10.1016/S00018686(01)00093-8

Sedjo, R. A., Lyon, K. S. (2015). The long-term adequacy of world timber supply: Routledge.

Shih, W. Y., Shih, W.-H., Aksay, I. A. (1999). Elastic and Yield Behavior of Strongly Flocculated Colloids. J Am Ceram, 82, 616-624. doi: 10.1111/j.1151-2916.1999.tb01809.x

Siró, I., Plackett, D. (2010). Microfibrillated cellulose and new nanocomposite materials: a review. Cellulose, 17, 459-494.

Slavin, J. L. (2005). Dietary fiber and body weight. Nutrition, 21, 411-418.

Tavernier, I., Wijaya, W., Van der Meeren, P., Dewettinck, K., Patel, A. R. (2016). Food-grade particles for emulsion stabilization. Trends in Food Sci Technol, 50, 159-174.

Trache, D., Hussin, M. H., Haafiz, M. M., Thakur, V. K. (2017). Recent progress in cellulose nanocrystals: sources and production. Nanoscale, 9, 1763-1786.

Winuprasith, T., Suphantharika, M. (2013). Microfibrillated cellulose from mangosteen (Garcinia mangostana L.) rind: Preparation, characterization, and evaluation as an emulsion stabilizer. Food Hydrocoll, 32, 383-394. doi: https://doi.org/10.1016/j.foodhyd.2013.01.023 


\section{Figures}
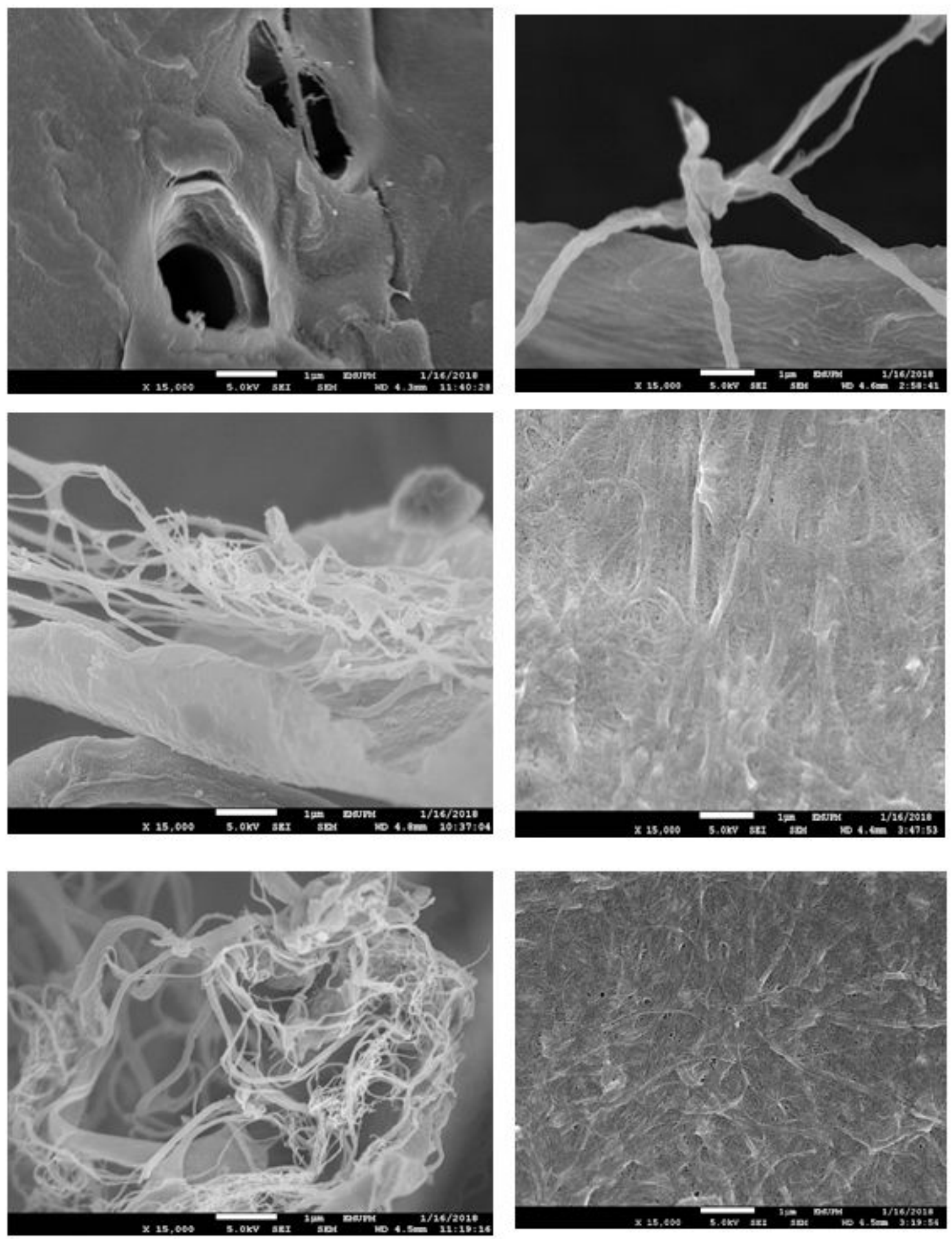

\section{Figure 1}

SEM photograph of MFC produced from different cycles of HPH. (A) Pretreated corn cob pulp-cycle 0, (B) cycle 2, (C) cycle 4, (D) cycle 6, (E) cycle 8 and (F) cycle 10 
A

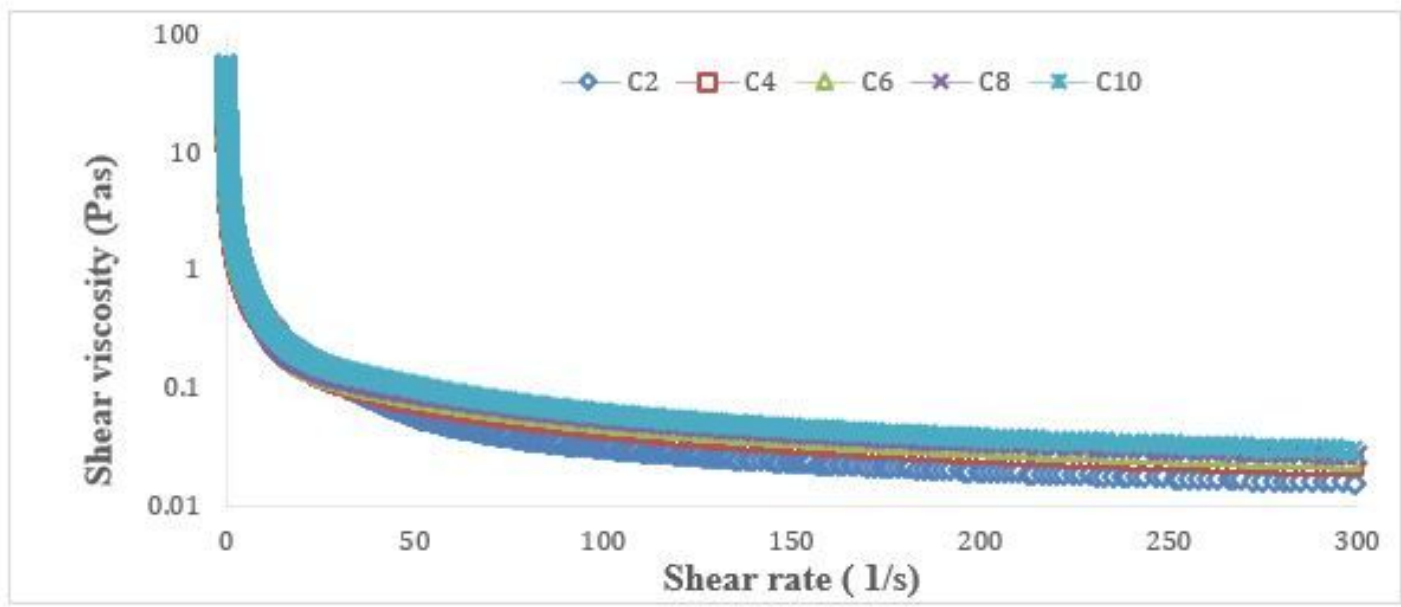

B

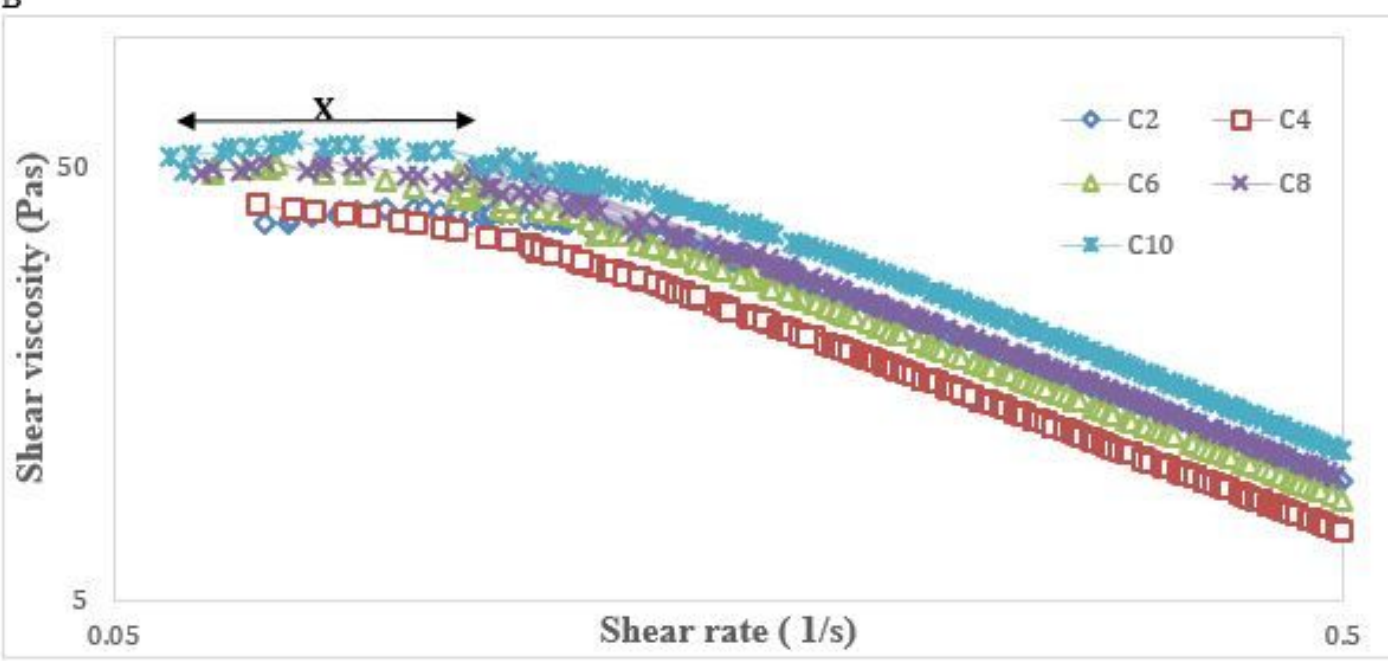

C

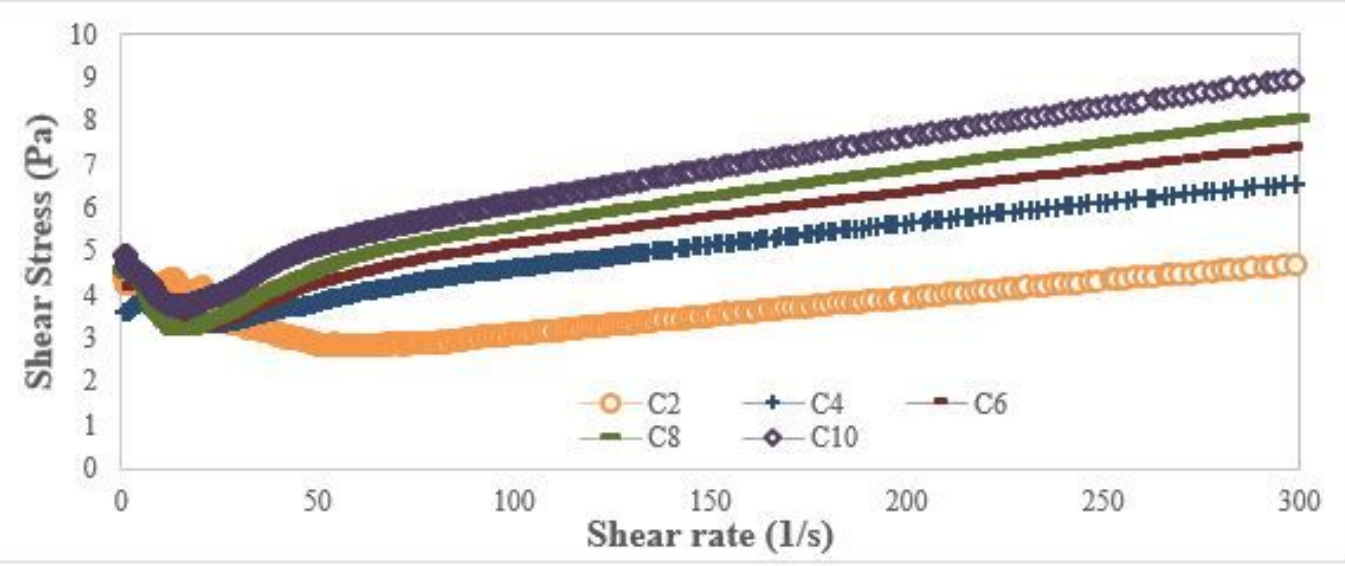

\section{Figure 2}

Flow properties of MFC from different cycles of HPH. (A) shear stress as a function of shear rate, (B) shear stress as a function of shear rate log scale (C) Shear stress as function of shear rate. $\mathrm{CO}$ represented cycle $0, \mathrm{C} 2$ represented cycle 2 , C4 represented cycle 4, C6 represented cycle 6, C8 represented cycle 8, C10 represented cycle 10 
A

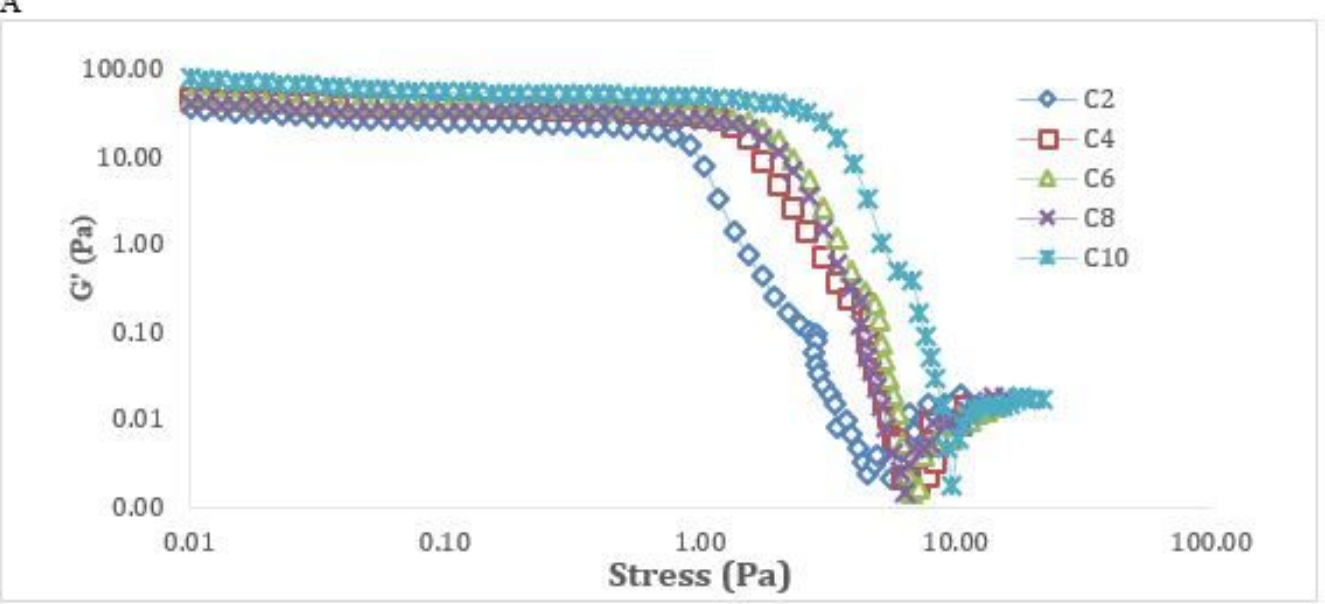

B

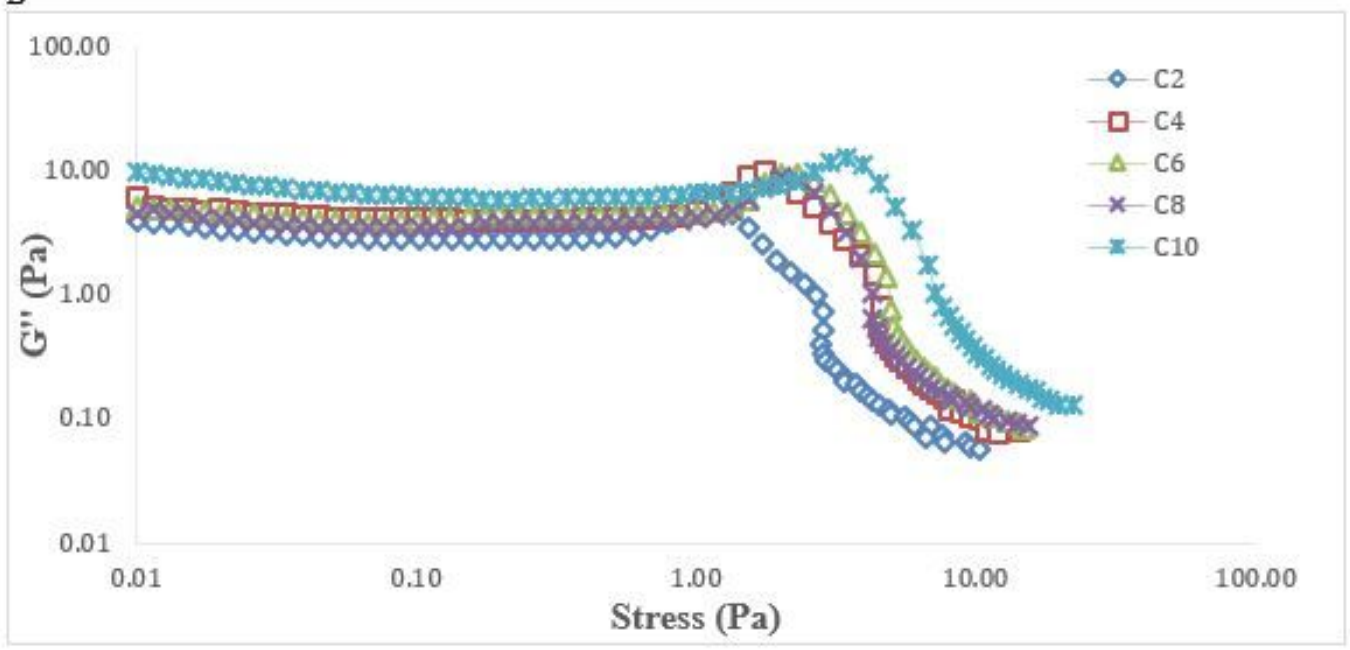

C

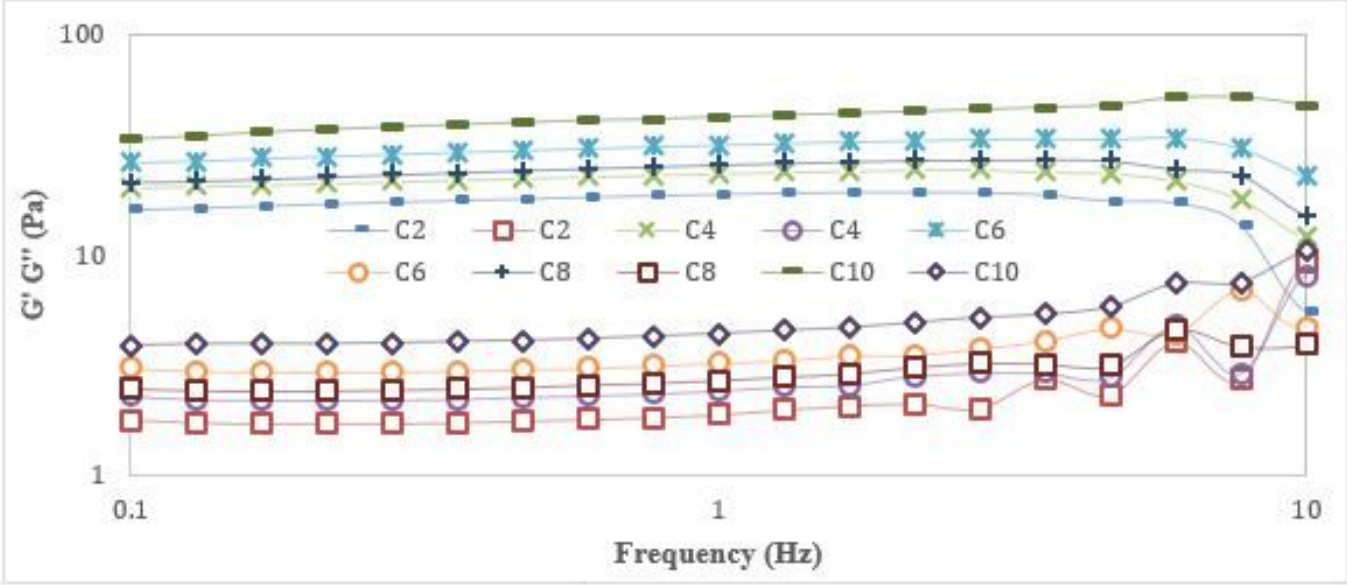

Figure 3

Viscoelastic properties of MFC produced from different cycles of HPH (A) storage modulus G' as a function of stress, (B) loss modulus as a function of stress log scale (C) G' (closed symbol) G" (open symbol) as function of frequency. C0 represented cycle 0, C2 represented cycle 2, C4 represented cycle 4, C6 represented cycle 6, C8 represented cycle 8, C10 represented cycle 10. 


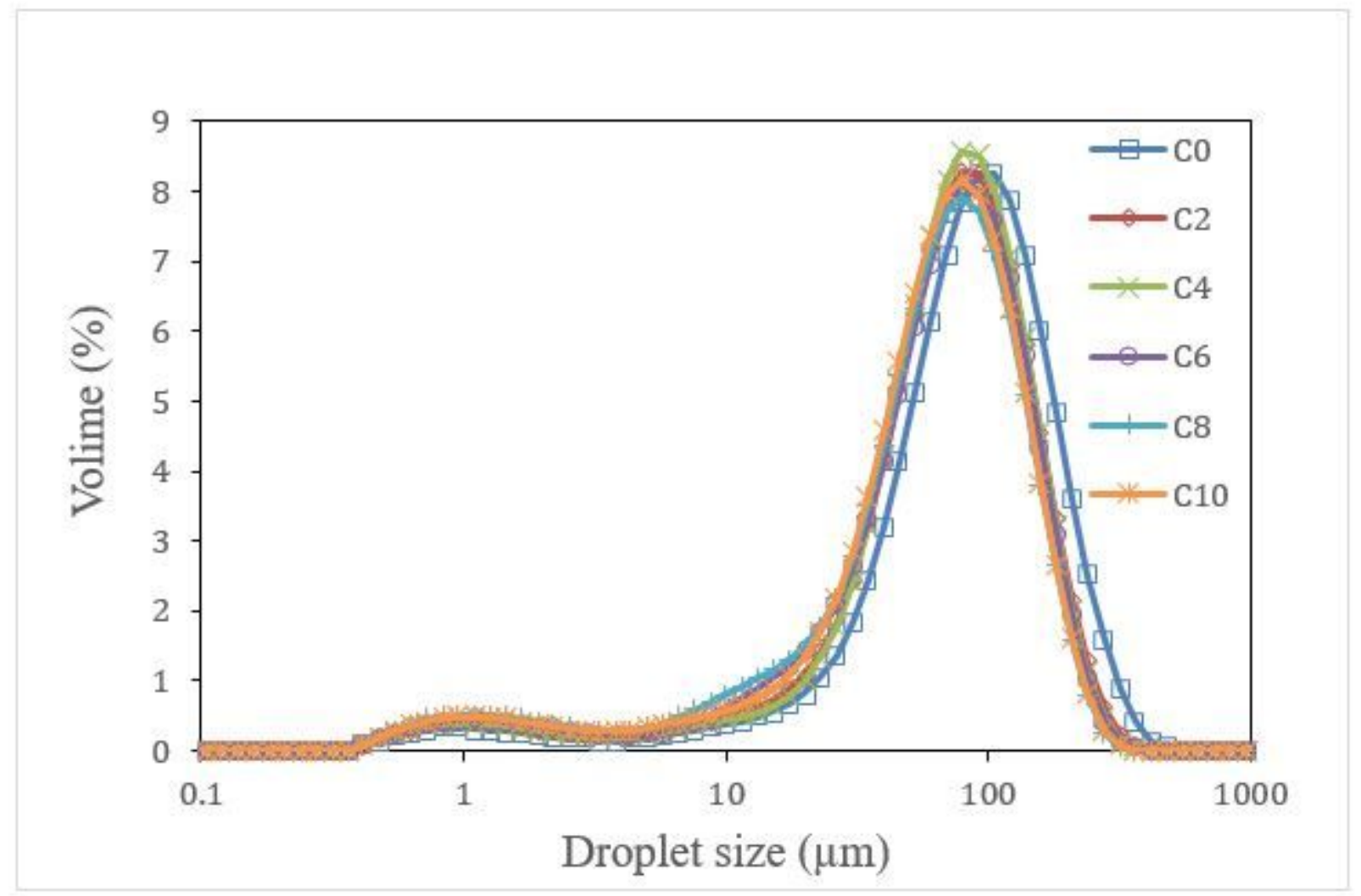

Figure 4

Particle size distributions of oil-in-water emulsions stabilized by microfibrillated celluloses prepared from different cycles of homogenizer. (C0 represented cycle 0, C2 represented cycle 2, C4 represented cycle 4, C6 represented cycle 6, C8 represented cycle 8, C10 represented cycle 10) 


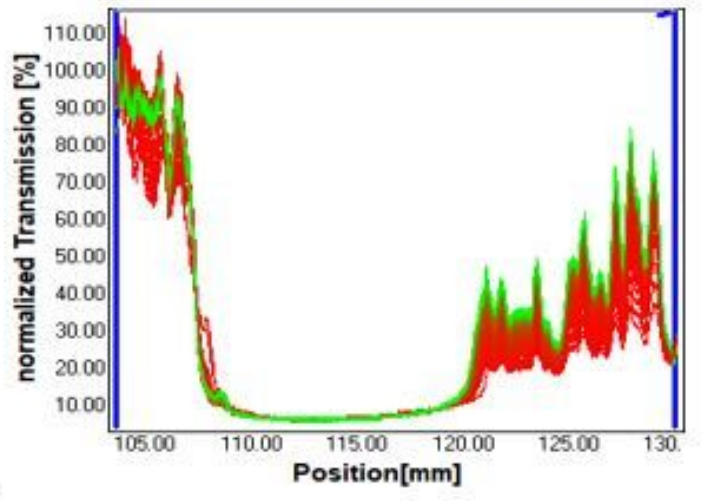

A

B

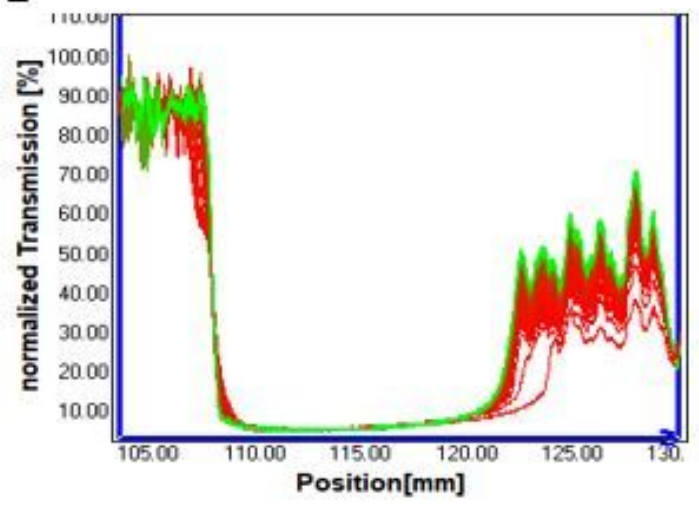

C

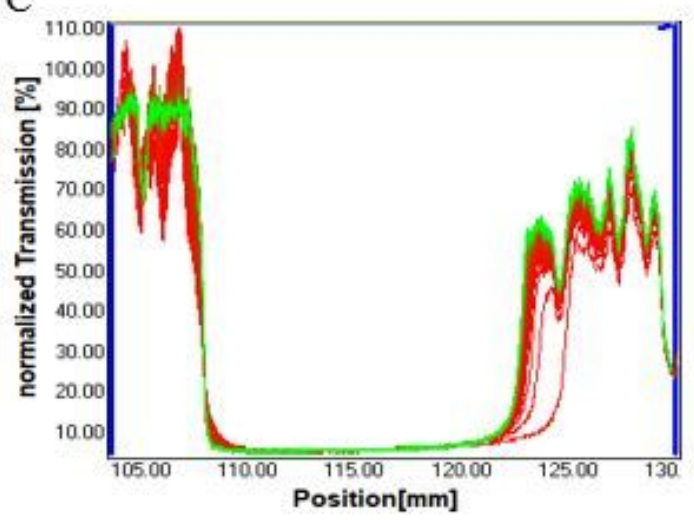

D

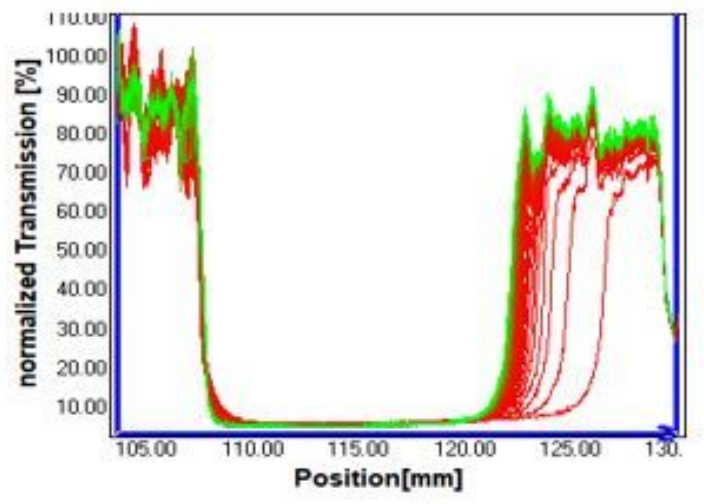

E

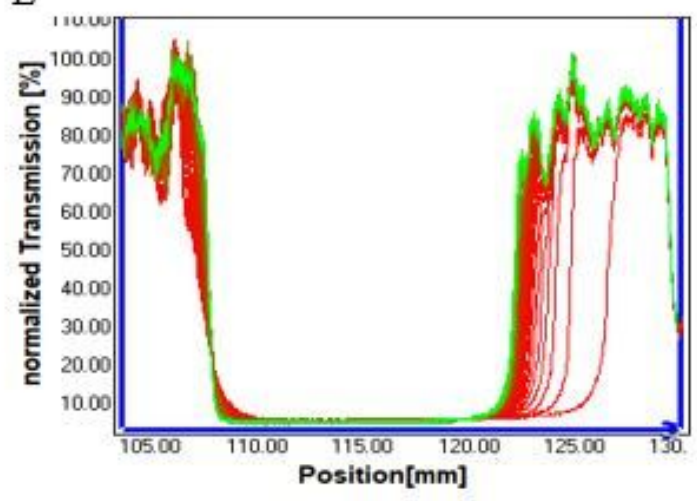

F

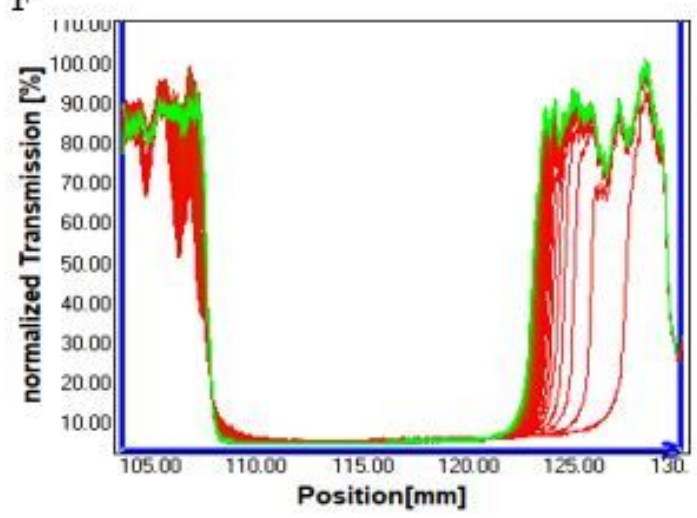

\section{Figure 5}

Lumifuge transmission profile over time of emulsions prepared using MFC from different cycles of high pressure homogenization at $25^{\circ} \mathrm{C}$. (A) cycle $0,(\mathrm{~B})$ cycle $2,(\mathrm{C})$ cycle 4 (D) cycle 6 (E) cycle 8 (F) cycle 10. Photograph of emulsion using MFC cycle 4 and cycle 10 at $25^{\circ} \mathrm{C}$. 
A
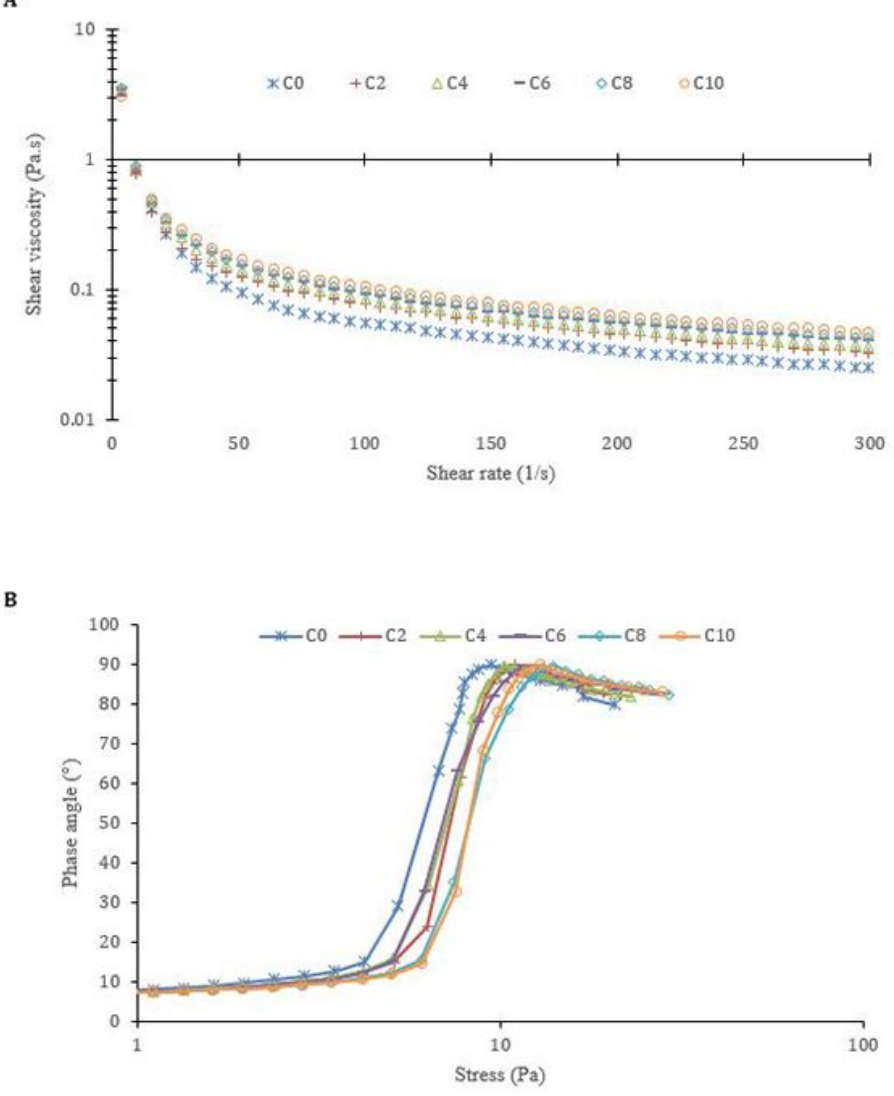

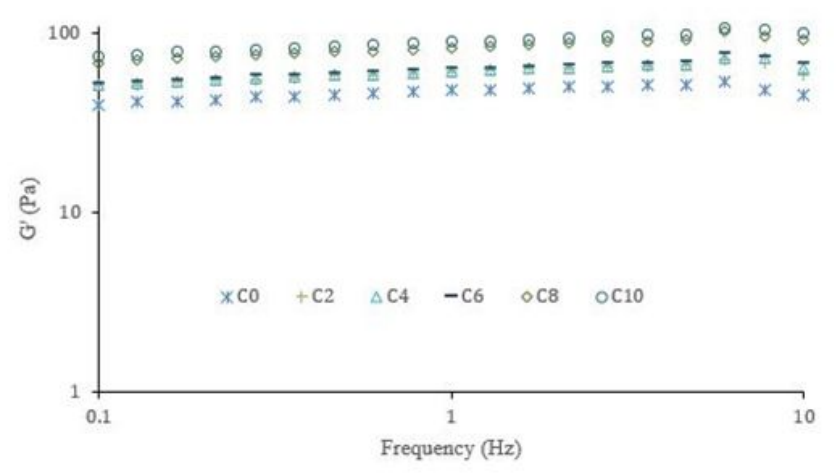

D

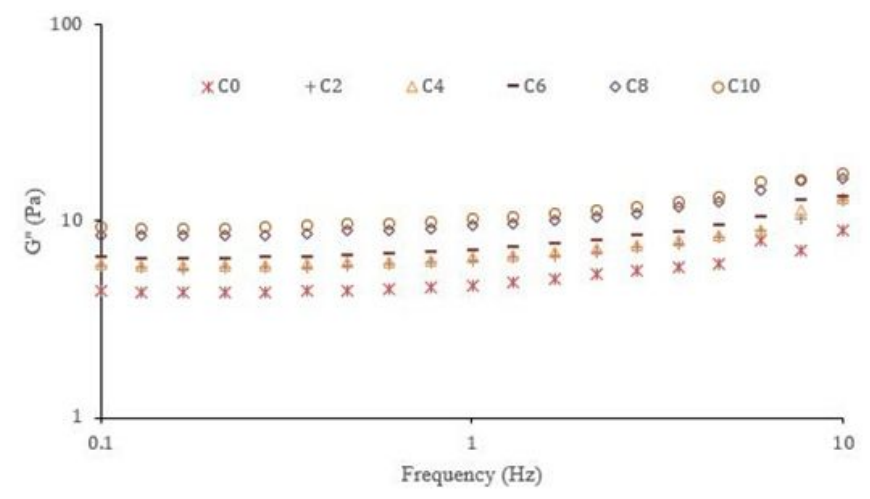

Figure 6

Rheological behavior of emulsions produced using different cycles of microfibrillated cellulose. (A) shear stress as a function of shear rate, (B) phase angle as a function shear stress (C) storage modulus $\mathrm{G}^{\prime}$ and (D) loss modulus G" as a function of angular frequency measured at temperature of $25^{\circ} \mathrm{C}$. The $\mathrm{C} 0$ represented emulsion produced using MFC from homogenization cycle 0 , so on and so forth. The final MFC amount in the emulsions were $0.45 \%$. 


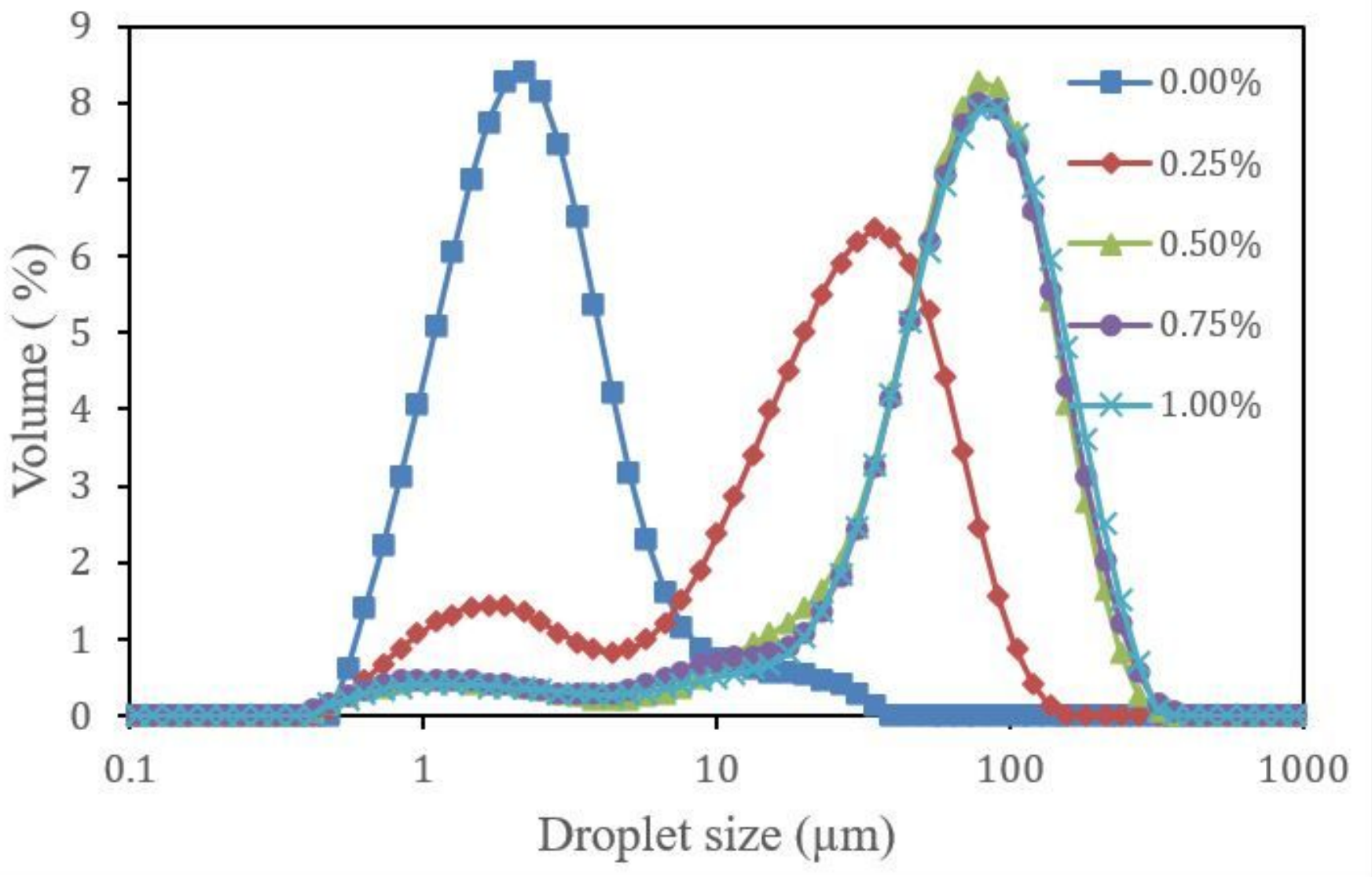

Figure 7

Particle size distributions of oil-in-water emulsions stabilized by $0 \%, 0.25 \%, 0.50 \%, 0,75 \%, 1.00 \%$ amount of microfibrillated celluloses. The final MFC amount in the emulsions were $0.45 \%$ in the cycle study. MFC used in the amount study were from high pressure homogenization cycle 10. 
A
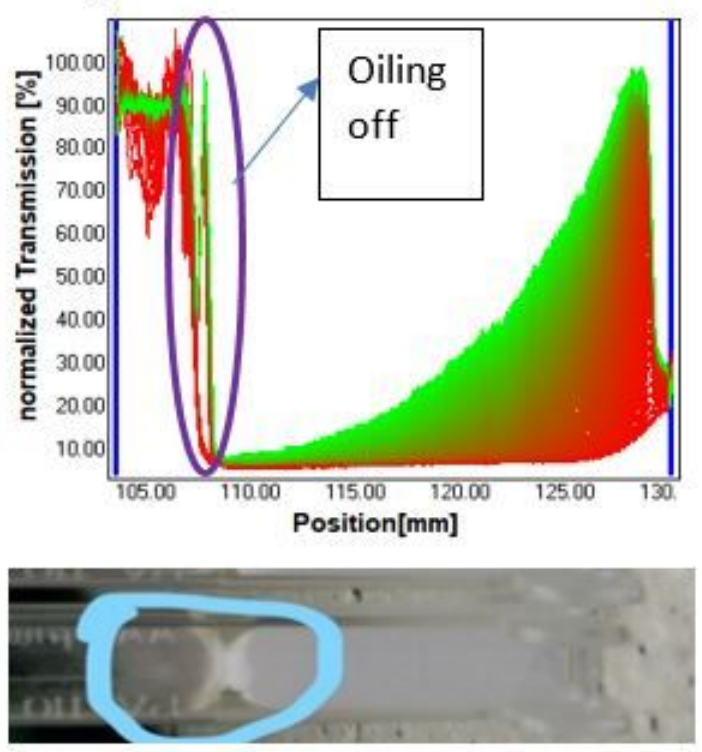

B
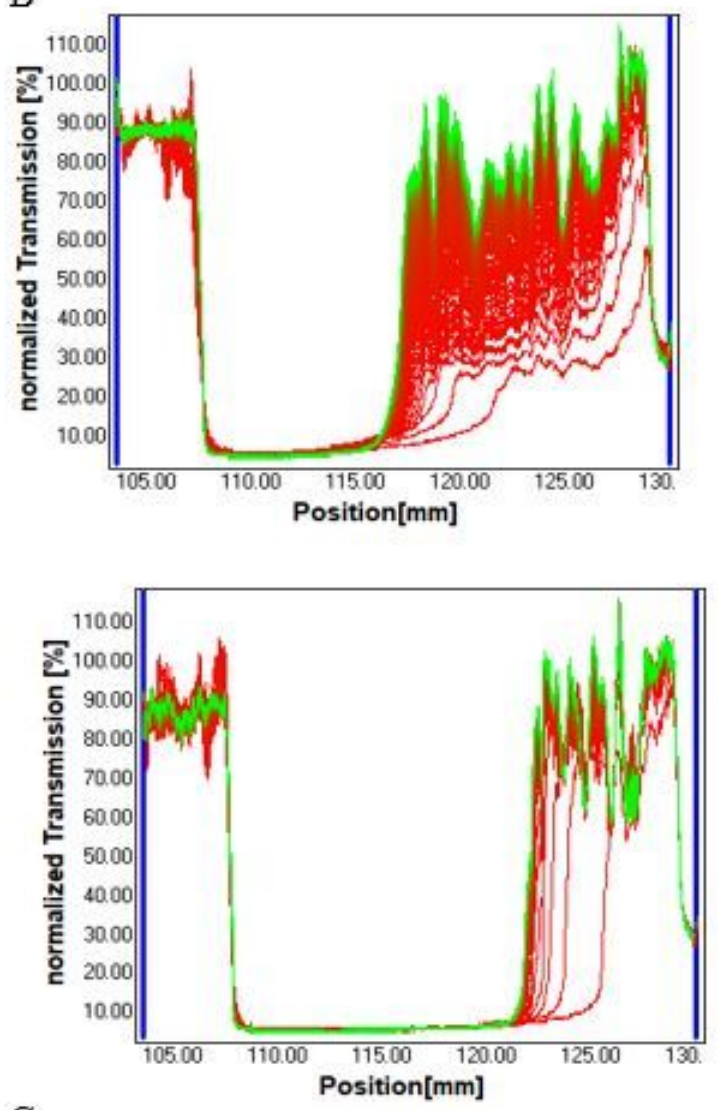

$\mathrm{D}$
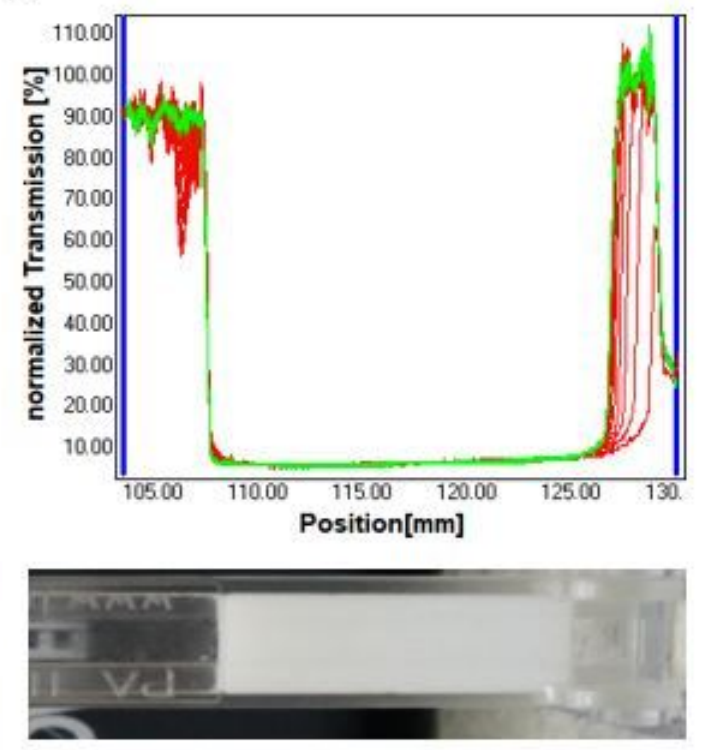

E
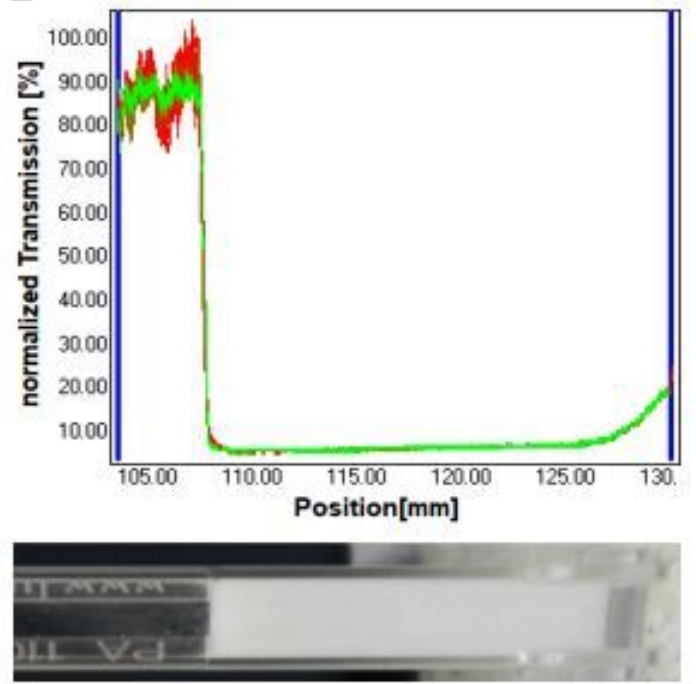

$\mathrm{C}$

\section{Figure 8}

Lumifuge transmission profile over time for emulsions stabilized prepared with different final amounts of MFC at $25^{\circ} \mathrm{C}$. (A) $0.00 \%$, (B) $0.25 \%$, (C) $0.5 \%$ (D) $0.75 \%$ and (E) $1.00 \%$ MFC with (A), (D) and (E) having photo of emulsions containing tubes with its respective final amount of MFC. 
A
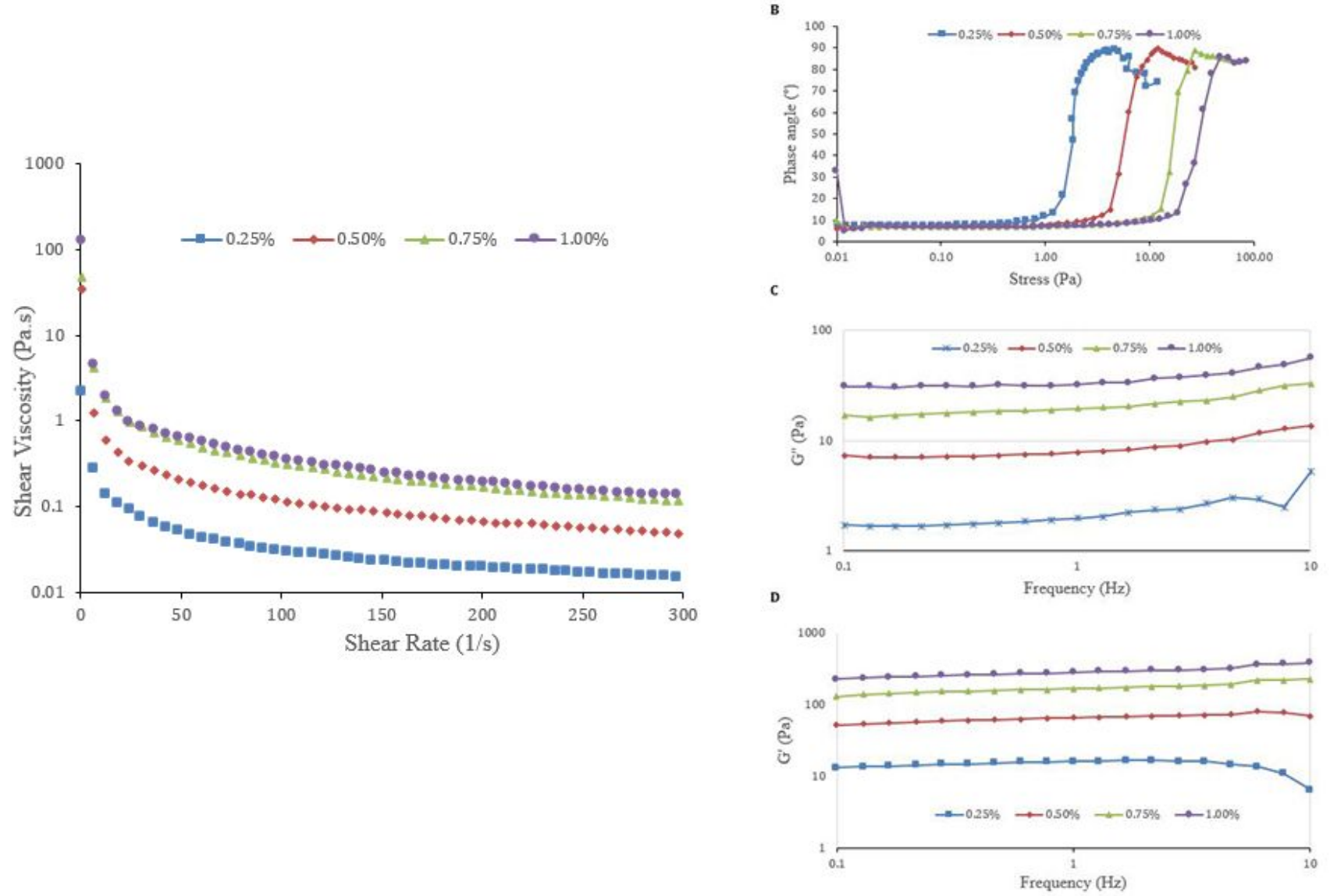

\section{Figure 9}

Effect of different amounts of microfibrillated cellulose on the rheological behavior of emulsions. The amount of MFC refers to the final amount of MFC in the emulsion and the MFC used were cycle 10. (A) shear stress as a function of shear rate, (B) phase angle as a function shear stress (C) storage modulus $\mathrm{G}^{\prime}$ and (D) loss modulus $\mathrm{G}^{\prime \prime}$ as a function of angular frequency measured at temperature of $25^{\circ} \mathrm{C}$.
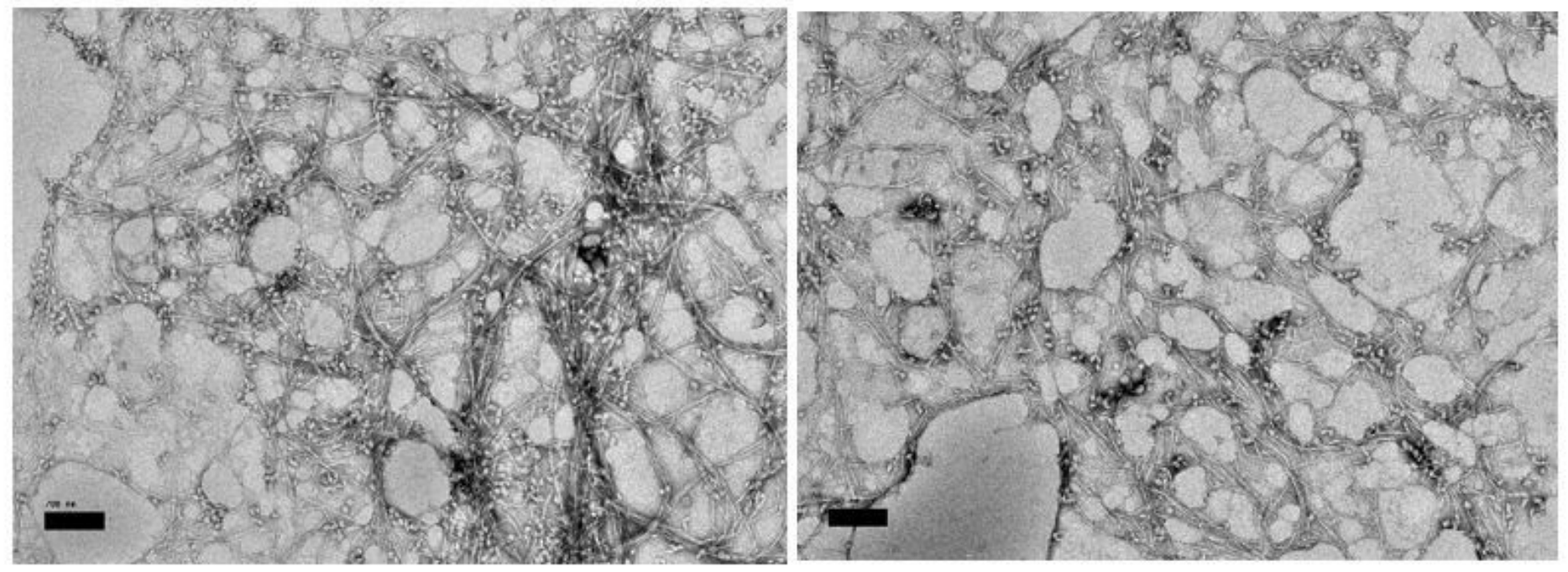
Morphologies of the microfibrillated cellulose-stabilized oil-in-water emulsions prepared using MFC produced from high pressure homogenization cycle 2 (left) and 10 (right) when observed under field emission analytical electron microscope with magnification power of 10000x. 